\title{
Branching rules in the ring of superclass functions of unipotent upper-triangular matrices
}

\author{
Nathaniel Thiem
}

Received: 20 November 2008 / Accepted: 2 June 2009 / Published online: 25 June 2009

(C) Springer Science+Business Media, LLC 2009

\begin{abstract}
It is becoming increasingly clear that the supercharacter theory of the finite group of unipotent upper-triangular matrices has a rich combinatorial structure built on set-partitions that is analogous to the partition combinatorics of the classical representation theory of the symmetric group. This paper begins by exploring a connection to the ring of symmetric functions in non-commuting variables that mirrors the symmetric group's relationship with the ring of symmetric functions. It then also investigates some of the representation theoretic structure constants arising from the restriction, tensor products and superinduction of supercharacters in this context.
\end{abstract}

Keywords Supercharacters $\cdot$ Set-partitions $\cdot$ Noncommutative symmetric functions · Finite unipotent groups

\section{Introduction}

The representation theory of the symmetric group $S_{n}$, with its connections to partition and tableaux combinatorics, has become a fundamental model in combinatorial representation theory. It has become clear in recent years that the representation theory of the finite group of unipotent upper-triangular matrix groups $U_{n}(q)$ can lead to a similarly rich combinatorial theory. While understanding the usual representation theory of $U_{n}(q)$ is a wild problem, André [1-4] and Yan [22, 23] constructed a natural approximation to the representation theory that leads to a more computable theory. This approximation (known as a super-representation theory) now relies on set-partition combinatorics in the same way that the representation theory of the symmetric group relies on partition combinatorics.

\footnotetext{
N. Thiem $(\bowtie)$

Department of Mathematics, University of Colorado at Boulder, Boulder, USA

e-mail: thiemn@colorado.edu
} 
A fundamental tool in symmetric group combinatorics is the ring of symmetric functions, which encodes the character theory of all symmetric groups simultaneously in a way that polynomial multiplication in the ring of symmetric functions becomes symmetric group induction from Young subgroups. This kind of a relationship has been extended to wreath products and type $A$ finite groups of Lie type (for descriptions see for example [15, 20]). One of the purposes of this paper is to suggest an analogous relationship between the supercharacter theory of $U_{n}(q)$ and the ring on symmetric functions in non-commuting variables NCSym. In particular, Corollary 3.2 shows that there are a family of algebra isomorphisms from the ring of supercharacters to NCSym, where we replace induction from subgroups with its natural analogue superinduction from subgroups. Unfortunately, there does not yet seem to be a canonical choice (ideally, such a choice would take the Hopf structure of NCSym into account).

The other purpose of this paper is to use the combinatorics of set partitions to supply recursive algorithms for computing restrictions to a family of subgroups called parabolic subgroups. It turns out that if $k \leq n$, then there are many ways in which $U_{k}(q)$ sits inside $U_{n}(q)$ as a subgroup. In fact, for every subset $A \subseteq\{1,2, \ldots, n\}$ with $k$ elements, there is a distinct subgroup $U_{A}$ of $U_{n}(q)$ isomorphic to $U_{k}(q)$. The restriction from $U_{n}(q)$ to $U_{A}$ depends on $A$, and Theorem 4.6 sorts out the combinatorial differences for all possible subsets $A$. This result can then be easily extended to give restriction rules for all parabolic subgroups. These computations require knowledge of tensor product results that were previously done by André [1] for large primes and by Yan [22] for arbitrary primes. For completeness, this paper supplies an alternate proof that relates tensor products to restriction and a generalization of the inflation functor (see Lemma 4.8).

By Frobenius reciprocity we then also obtain the coefficients of superinduction from these subgroups. Corollary 4.14 concludes by observing that superinduced supercharacters from parabolic subgroups are essentially twisted super-permutation characters (again using the generalization of the inflation map). These results give the structure constants for the ring of superclass functions of the finite unipotent uppertriangular groups. However, the underlying coefficient ring is $\mathbb{Z}\left[q^{-1}\right]$, unlike in the case of the symmetric group where the ring is $\mathbb{Z}$.

The paper is organized as follows. Section 2 introduces some set-partition combinatorics, describes the parabolic subgroups that will replace Young subgroups in our theory, reviews the supercharacter theory of pattern groups (as defined in [10]), and recalls the ring of symmetric functions in non-commuting variables NCSym. We proceed in Section 3 by describing the family of isomorphisms between NCSym and the ring of supercharacters. Section 4 uses the fact that supercharacters of $U_{n}(q)$ decompose into tensor products of simpler characters to supply algorithms for computing restrictions and superinductions of supercharacters. These results generalize restriction results in [20], and make use of a new generalization of the inflation functor to supercharacters of pattern groups.

This paper builds on [16] and [19] by giving restriction and superinduction formulas for larger families of groups. These formulas are computable, and have been implemented in Python as part of an honors thesis at the University of Colorado [14]. Other recent work in this area worth mentioning includes extensions by André and 
his collaborators to supercharacter theories of other types [5] and over other rings [6], explorations of all supercharacter theories for a given group by Hendrickson in his thesis [13], and an intriguing unexplored connection to $L$-packets in the work of Drinfeld and Boyarchenko [9].

\section{Preliminaries}

This section reviews the combinatorics needed for the main results, gives a brief introduction to the supercharacter theory of pattern groups, and recalls the ring of symmetric functions in non-commuting variables.

$2.1 \mathbb{F}_{q}$-labeled set-partitions

For $A \subseteq\{1,2, \ldots, n\}$, let

$$
\mathcal{S}_{A}=\{\text { set-partitions of } A\}
$$

and

$$
\mathcal{S}=\bigcup_{n \geq 0} \mathcal{S}_{n}, \quad \text { where } \quad \mathcal{S}_{n}=\mathcal{S}_{\{1,2, \ldots, n\}} .
$$

An $\operatorname{arc} i \frown j$ of $K \in \mathcal{S}_{A}$ is a pair $(i, j) \in A \times A$ such that

(1) $i<j$,

(2) $i$ and $j$ are in the same part of $K$,

(3) if $k$ is in the same part as $i$ and $i<k \leq j$, then $k=j$.

Thus, if we order each part in increasing order, then the arcs are pairs of adjacent elements in each part. For example,

$$
\{1,5,7\} \cup\{2,3\} \cup\{4\} \cup\{6,8,9\} \in \mathcal{S}_{9}
$$

has $\operatorname{arcs} 1 \frown 5,5 \frown 7,2 \frown 3,6 \frown 8$, and $8 \frown 9$. We can also represent the set partition $K$ as a diagram consisting of $|K|$ vertices with an edge connecting vertex $i$ to vertex $j$ if $i \frown j$ is an $\operatorname{arc}$ of $K$; for example,

$$
\{1,5,7\} \cup\{2,3\} \cup\{4\} \cup\{6,8,9\}
$$

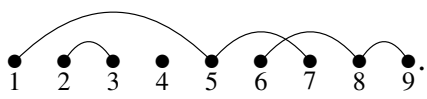

The $\operatorname{arcset} \mathcal{A}(K)$ of $K \in \mathcal{S}_{A}$ is

$$
\mathcal{A}(K)=\{\operatorname{arcs} \text { of } K\} \text {. }
$$

A crossing of $K \in \mathcal{S}_{A}$ is a pair of $\operatorname{arcs}(i \frown k, j \frown l) \in \mathcal{A}(K) \times \mathcal{A}(K)$ such that $i<j<k<l$. The crossing set $C(K)$ of $K$ is

$$
C(K)=\{\text { crossings of } K\} \text {. }
$$

For example, if $K=\{1,5,7\} \cup\{2,3\} \cup\{4\} \cup\{6,8,9\}$, then $K$ has one crossing $(5 \frown$ $7,6 \frown 8)$, as is easily observed in the above diagrammatic representation of $K$. 
An $\mathbb{F}_{q}$-labeled set-partition of $A$ is a pair $\left(\lambda, \tau_{\lambda}\right)$, where $\lambda$ is a set-partition of $A$ and $\tau_{\lambda}: \mathcal{A}(\lambda) \rightarrow \mathbb{F}_{q}^{\times}$is a labeling of the arcs by nonzero elements of $\mathbb{F}_{q}$. By convention, if $\tau_{\lambda}(i \frown j)=a$, then we write the arc as $i \stackrel{a}{\sim} j$. Thus, we can typically suppress the labeling function in the notation. Let

$$
\mathcal{S}_{A}(q)=\left\{\mathbb{F}_{q} \text {-labeled set-partitions of } A\right\},
$$

and

$$
\mathcal{S}(q)=\bigcup_{n \geq 0} \mathcal{S}_{n}(q), \quad \text { where } \quad \mathcal{S}_{n}(q)=\mathcal{S}_{\{1,2, \ldots, n\}}(q) .
$$

Note that if $s_{n}(q)=\left|\mathcal{S}_{n}(q)\right|$, then the generating function

$$
\sum_{n \geq 0} s_{n}(q) \frac{x^{n}}{n !}=e^{\frac{e^{(q-1) x}-1}{q-1}}
$$

is a $q$-analogue of the usual exponential generating function of the Bell numbers (where $q=2$ gives the usual generating function [18]).

Suppose $A \subseteq B \subseteq\{1,2, \ldots, n\}$. Then there is a function

$$
\begin{aligned}
\langle\cdot\rangle_{B}:\left\{\begin{array}{c}
\mathbb{F}_{q} \text {-labeled } \\
\text { set-partitions of } A
\end{array}\right\} & \longrightarrow\left\{\begin{array}{c}
\mathbb{F}_{q} \text {-labeled } \\
\text { set-partitions of } B
\end{array}\right\} \\
\lambda & \mapsto\langle\lambda\rangle_{B}
\end{aligned}
$$

where $\langle\lambda\rangle_{B}$ is the unique $\mathbb{F}_{q}$-labeled set-partition of $B$ with arc set $\mathcal{A}(\lambda)$ and labeling function $\tau_{\lambda}$. We will use the convention that $\langle\lambda\rangle_{n}=\langle\lambda\rangle_{\{1,2, \ldots, n\}}$. For example, if $A=$ $\{2,3,5,7\}, a, b \in \mathbb{F}_{q}^{\times}$, and

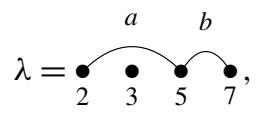

then

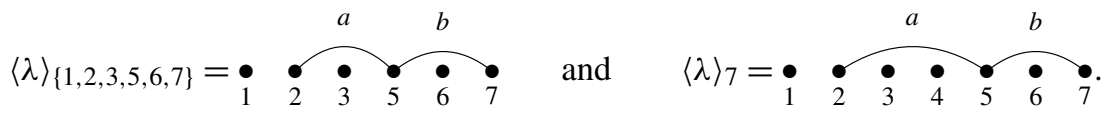

Because the relative position of $A \subseteq\{1,2, \ldots, n\}$ is critical, we will sometimes indicate the elements of $A^{c}$ in the diagrams of $\mathcal{S}_{A}(q)$ by replacing $\bullet$ by ०. For example, if $A=\{2,3,5,7\}$ and

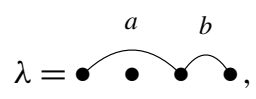

then to indicate what numbers are in $A$, we write

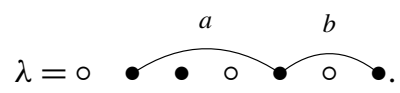




\subsection{Pattern groups}

For $n \in \mathbb{Z}_{\geq 1}$, let $U_{n}(q)$ be the group of $n \times n$ unipotent upper-triangular matrices with entries in $\overline{\mathbb{F}}_{q}$. Given a poset $\mathcal{P}$ of $\{1,2, \ldots, n\}$, the pattern group $U_{\mathcal{P}}(q)$ is

$$
U_{\mathcal{P}}(q)=\left\{u \in U_{n}(q) \mid u_{i j} \neq 0 \text { implies } i \preceq j \text { in } \mathcal{P}\right\} .
$$

Remark If $T_{n}(q)$ is the group of $n \times n$ diagonal matrices with entries in $\mathbb{F}_{q}^{\times}$, then the set of pattern subgroups of $U_{n}(q)$ can be characterized as the set of subgroups fixed by the conjugation action of $T_{n}(q)$ on $U_{n}(q)$. In particular, this observation implies pattern groups are indeed groups. More directly, note that closure in $U_{\mathcal{P}}(q)$ follows from the transitivity of the poset $\mathcal{P}$.

Consider the injective map

$$
\begin{aligned}
& \mathcal{S}_{n} \longrightarrow\left\{\begin{array}{c}
\text { Posets of } \\
\{1,2, \ldots, n\}
\end{array}\right\} \\
& K \mapsto \mathcal{P}_{K}
\end{aligned}
$$

where $i \prec j$ in $\mathcal{P}_{K}$ if and only if $i<j$ and both $i$ and $j$ are in the same part of $K$.

A pattern subgroup $U_{\mathcal{P}}(q)$ is a parabolic subgroup of $U_{n}(q)$ if there exists $K \in \mathcal{S}_{n}$ such that $\mathcal{P}=\mathcal{P}_{K}$. Note that if $K=K_{1} \cup K_{2} \cup \cdots \cup K_{\ell}$ is the decomposition of $K$ into parts, then

$$
U_{\mathcal{P}_{K}}(q) \cong U_{\left|K_{1}\right|}(q) \times U_{\left|K_{2}\right|}(q) \times \cdots \times U_{\left|K_{\ell}\right|}(q) .
$$

Thus, the parabolic subgroups of $U_{\mathcal{P}}(q)$ are reminiscent of the Young subgroups of the symmetric groups $S_{n}$ or parabolic subgroups of the general linear groups $\operatorname{GL}_{n}(q)$. In fact, we will follow this analogy into the supercharacter theory of $U_{n}(q)$. To simplify notation, we will typically write

$$
U_{K}(q)=U_{\mathcal{P}_{K}}(q), \quad \text { for } K \in \mathcal{S}_{n} .
$$

\section{Remarks}

(a) These subgroups are not generally block diagonal. For example,

$$
U_{\mathcal{P}_{\{1,3,5\} \cup\{2,4\}}}=\left\{\left(\begin{array}{ccccc}
1 & 0 & * & 0 & * \\
0 & 1 & 0 & * & 0 \\
0 & 0 & 1 & 0 & * \\
0 & 0 & 0 & 1 & 0 \\
0 & 0 & 0 & 0 & 1
\end{array}\right) \mid * \in \mathbb{F}_{q}\right\} \cong U_{3}(q) \times U_{2}(q) .
$$

(b) Parabolic subgroups do not include all possible copies of pattern subgroups isomorphic to a direct product of $U_{k}(q)$ 's. For example,

$$
U \underbrace{4}_{1}=\left\{\left(\begin{array}{llll}
1 & 0 & * & * \\
0 & 1 & 0 & * \\
0 & 0 & 1 & * \\
0 & 0 & 0 & 1
\end{array}\right) \mid * \in \mathbb{F}_{q}\right\} \cong U_{3}(q) \times U_{2}(q)
$$

is not a parabolic subgroup of $U_{4}(q)$. 


\subsection{A supercharacter theory for pattern groups}

Given a group $G$, a supercharacter theory is an approximation to the usual character theory. To be more precise, a supercharacter theory consists of a set of superclasses $\mathcal{K}$ and a set of supercharacters $\mathcal{X}$, such that

(a) the set $\mathcal{K}$ is a partition of $G$ such that each part is a union of conjugacy classes,

(b) the set $\mathcal{X}$ is a set of characters such that each irreducible character appears as the constituent of exactly one supercharacter,

(c) the supercharacters are constant on superclasses,

(d) $|\mathcal{K}|=|\mathcal{X}|$

(e) the identity element 1 of $G$ is in its own superclass, and the trivial character $\mathbb{1}$ of $G$ is a supercharacter.

This general notion of a supercharacter theory was introduced by Diaconis and Isaacs [10] to generalize work of André and Yan on the character theory of $U_{n}(q)$.

Remark The definition includes a reasonable amount of redundancy, as explored in $[10,13]$.

Diaconis and Isaacs extended the construction of André of a supercharacter theory for $U_{n}(q)$ to a larger family of groups called algebra groups. We will review the construction for pattern groups (a subset of the set of algebra groups). Let $\mathcal{P}$ be a poset of $\{1,2, \ldots, n\}$ and let

$$
\mathfrak{n}_{\mathcal{P}}(q)=U_{\mathcal{P}}(q)-1,
$$

which is an $\mathbb{F}_{q}$-algebra (for $u \in U_{\mathcal{P}}(q)$, the matrix $u-1$ is the matrix $u$ with the ones on the diagonal replaced by zeroes).

Fix a nontrivial homomorphism $\vartheta: \mathbb{F}_{q}^{+} \rightarrow \mathbb{C}^{\times}$. The pattern group $U_{\mathcal{P}}(q)$ acts on the left and right on both $\mathfrak{n}_{\mathcal{P}}(q)$ and the dual space $\mathfrak{n}_{\mathcal{P}}(q)^{*}$, and the two-sided orbits lead to the sets $\mathcal{K}$ and $\mathcal{X}$ by the following rules. The superclasses are given by

$$
\begin{aligned}
U_{\mathcal{P}}(q) \backslash \mathfrak{n}_{\mathcal{P}}(q) / U_{\mathcal{P}}(q) & \longleftrightarrow \mathcal{K} \\
U_{\mathcal{P}}(q) X U_{\mathcal{P}}(q) & \mapsto 1+U_{\mathcal{P}}(q) X U_{\mathcal{P}}(q),
\end{aligned}
$$

and the supercharacters are given by

$$
\begin{aligned}
U_{\mathcal{P}}(q) \backslash \mathfrak{n}_{\mathcal{P}}(q)^{*} / U_{\mathcal{P}}(q) & \longleftrightarrow \mathcal{X} \\
U_{\mathcal{P}}(q) \lambda U_{\mathcal{P}}(q) & \mapsto \chi^{\lambda}=\frac{\left|\lambda U_{\mathcal{P}}(q)\right|}{\left|U_{\mathcal{P}}(q) \lambda U_{\mathcal{P}}(q)\right|} \sum_{\mu \in U_{\mathcal{P}}(q) \lambda U_{\mathcal{P}}(q)} \vartheta \circ \mu .
\end{aligned}
$$

The corresponding $U_{\mathcal{P}}(q)$-modules are given by

$$
V^{\lambda}=\mathbb{C}-\operatorname{span}\left\{v_{\mu} \mid \mu \in U_{\mathcal{P}}(q) \lambda\right\},
$$

with action

$$
g v_{\mu}=\vartheta((g \mu)(1-g)) v_{g \mu}, \quad \text { for } g \in U_{\mathcal{P}}(q) \text { and } \mu \in U_{\mathcal{P}}(q) \lambda .
$$


Examples The group $U_{n}(q)$ was the original motivation for studying supercharacter theories. The following results are due to André, Yan, and Arias-Castro-DiaconisStanley. The number of superclasses is

$$
|\mathcal{K}|=|\mathcal{X}|=\left|\mathcal{S}_{n}(q)\right|,
$$

where, for example,

$$
\begin{aligned}
\mathcal{S}_{n}(q) & \longrightarrow \mathcal{K} \\
\mu & \mapsto u_{\mu},
\end{aligned} \quad \text { and } \quad\left(u_{\mu}\right)_{i j}= \begin{cases}1, & \text { if } i=j, \\
\tau_{\mu}(i \frown j), & \text { if } i \frown j \in \mathcal{A}(\mu), \\
0, & \text { otherwise. }\end{cases}
$$

The corresponding supercharacter formula for $\lambda, \mu \in \mathcal{S}_{n}(q)$ is

$$
\chi^{\lambda}\left(u_{\mu}\right)= \begin{cases}\prod_{i \frown l \in \mathcal{A}(\lambda)} \frac{q^{l-i-1} \vartheta\left(\tau_{\lambda}(i \frown l) \tau_{\mu}(i \frown l)\right)}{q^{|\{j \frown k \in \mathcal{A}(\mu) \mid i<j<k<l\}|},}, & \text { if } i<j<k, i \frown k \in \mathcal{A}(\lambda) \\ 0, & \text { implies } i \frown j, j \frown k \notin \mathcal{A}(\mu), \\ \text { otherwise, }\end{cases}
$$

where $\tau_{\mu}(i \frown l)=0$ if $i \frown l \notin \mathcal{A}(\mu)$ (see [11] for the corresponding formula for arbitrary pattern groups).

Remark We abuse notation slightly by identifying $\lambda \in \mathcal{P}_{n}(q)$ with the element $\lambda \in \mathfrak{n}_{n}^{*}$ given by

$$
\lambda(u-1)=\sum_{i \stackrel{a}{\sim} j \in \mathcal{A}(\lambda)} a u_{i j} .
$$

Note that the degree of each character is

$$
\chi^{\lambda}(1)=\prod_{i \frown l \in \mathcal{A}(\lambda)} q^{l-i-1} .
$$

It follows directly from the formula that the supercharacters factor nicely

$$
\chi^{\lambda}=\prod_{i \stackrel{a}{=} l \in \mathcal{A}(\lambda)} \chi^{\langle i \stackrel{a}{-} l\rangle_{n}}
$$

It also follows from (2.1) and (2.2) that $\chi^{\lambda}$ is linear if and only if

$$
i \frown k \in \mathcal{A}(\lambda) \quad \text { implies } \quad k=i+1 .
$$

The set of crossings $C(\lambda)$ measures how close the supercharacter $\chi^{\lambda}$ is to being irreducible. In fact,

$$
\left\langle\chi^{\lambda}, \chi^{\mu}\right\rangle=q^{|C(\lambda)|} \delta_{\lambda \mu}
$$

where $\langle\cdot, \cdot\rangle$ is the usual inner product on characters.

For parabolics subgroups $U_{K}(q)$ of $U_{n}(q)$,

$$
|\mathcal{K}|=|\mathcal{X}|=\left|\mathcal{S}_{\left|K_{1}\right|}(q)\right|\left|\mathcal{S}_{\left|K_{2}\right|}(q)\right| \cdots\left|\mathcal{S}_{\left|K_{\ell}\right|}(q)\right|,
$$

where $K=K_{1} \cup K_{2} \cup \cdots \cup K_{\ell} \in \mathcal{S}_{n}$. 
Remark If instead of considering $U_{n}(q)$-orbits on $\mathfrak{n}_{n}(q)$ and $\mathfrak{n}_{n}(q)^{*}$, we consider orbits of the full Borel subgroup $B_{n}(q)=T_{n}(q) U_{n}(q)$ on these spaces, then the corresponding supercharacter theory no longer depends on the finite field $q$. Thus, the combinatorics reduces to considering set-partitions rather than $\mathbb{F}_{q}$-labeled setpartitions. In general, if $(\mathcal{K}, \mathcal{X})$ is any supercharacter theory of a finite group $G$, and $H \subseteq \operatorname{Aut}(G)$, then there exists a (potentially) new supercharacter theory of $G$ with superclasses given by $H$-orbits in $\mathcal{K}$ [10]. In this case, $T_{n}(q)$ acts on $\mathcal{S}_{n}(q)$ by $t\left(\lambda, \tau_{\lambda}\right)=\left(\lambda, t \circ \tau_{\lambda}\right)$, where $\lambda \in \mathcal{S}_{n}(q), t \in T_{n}(q)$ and $t \circ \tau_{\lambda}(i \frown j)=t_{i} \tau_{\lambda}(i \frown j) t_{j}^{-1}$, for $i \frown j \in \mathcal{A}(\lambda)$.

Supercharacters satisfy a variety of nice properties, as described in [10]. The above construction satisfies

(a) The product of two supercharacters is a $\mathbb{Z}_{\geq 0}$-linear combination of supercharacters.

(b) The restriction of a supercharacter from one pattern group to a pattern subgroup is a $\mathbb{Z}_{\geq 0}$-linear combination of supercharacters.

However, it is not true that the usual induction functor on class functions of finite groups sends a supercharacter to a $\mathbb{Z}_{\geq 0}$-linear combination of supercharacters. In fact, an induced supercharacter is generally no longer even a superclass function.

Diaconis and Isaacs therefore define a superinduction map on supercharacters that is adjoint to restriction with respect to the usual inner product on class functions; it turns out that this function averages over superclasses in the same way induction averages over conjugacy classes. In particular, if $H \subseteq G$ are pattern groups (or more generally algebra groups), then superinduction is the function

$$
\begin{aligned}
& \text { SInd : }\left\{\begin{array}{c}
\text { Superclass functions } \\
\text { of } H
\end{array}\right\} \rightarrow\left\{\begin{array}{c}
\text { Superclass functions } \\
\text { of } G
\end{array}\right\} \\
& \chi \mapsto \operatorname{SInd}_{H}^{G}(\chi),
\end{aligned}
$$

where

$$
\operatorname{SInd}_{H}^{G}(\chi)(g)=\frac{1}{|G||H|} \sum_{\substack{x, y \in G \\ x(g-1) y+1 \in H}} \chi(1+x(g-1) y), \quad \text { for } g \in G
$$

Unfortunately, while SInd sends superclass functions to superclass functions, it sends supercharacters to $\mathbb{Z}_{\geq 0}[1 / q]$-linear combinations of supercharacters (where $q$ comes from the underlying finite field). In fact, the image is not even generally a character. See also [16] for a further exploration of the relationship between superinduction and induction. 
2.4 The ring of symmetric functions in non-commutative variables

Fix a set $X=\left\{X_{1}, X_{2}, \ldots\right\}$ of countably many non-commuting variables. For $K=$ $K_{1} \cup K_{2} \cup \cdots \cup K_{\ell} \in \mathcal{S}_{n}$, define the monomial symmetric function

$$
m_{K}(X)=\sum_{\substack{k=\left(k_{1}, k_{2}, \ldots, k_{\ell}\right) \in \mathbb{Z}_{\geq 1}^{\ell} \\ k_{i} \neq k_{j}, 1 \leq i<j \leq \ell}} X_{\pi_{1}(k)} X_{\pi_{2}(k)} \cdots X_{\pi_{\ell}(k)}, \quad \text { where } \pi_{j}(k)=k_{i} \text { if } j \in K_{i}
$$

The space of symmetric functions in non-commuting variables of homogeneous degree $n$ is

$$
\operatorname{NCSym}_{n}(X)=\mathbb{C}-\operatorname{span}\left\{m_{K}(X) \mid K \in \mathcal{S}_{n}\right\},
$$

and the ring of symmetric functions in non-commuting variables is

$$
\mathrm{NCSym}=\bigoplus_{n \geq 0} \operatorname{NCSym}_{n}(X),
$$

where a possible multiplication is given by usual polynomial products. However, note that if $K=\left\{a_{1}<a_{2}<\cdots<a_{m}\right\} \cup\left\{b_{1}<b_{2}<\cdots<b_{n}\right\} \in \mathcal{S}_{m+n}$ with $w=$ $\left(a_{1}, a_{2}, \cdots, a_{k_{m}}, b_{1}, b_{2}, \ldots, b_{n}\right)$ the corresponding permutation of $m+n$ elements, then we could "shuffle" two words according to $K$,

$$
\left(X_{i_{1}} X_{i_{2}} \cdots X_{i_{m}}\right) *_{K}\left(X_{i_{m+1}} \cdots X_{i_{m+n}}\right)=X_{i_{w^{-1}(1)}} X_{i_{w^{-1}(2)}} \cdots X_{i_{w^{-1}(m+n)}}
$$

These operations give a variety of alternate shuffle products for NCSym.

Remark Note that $\operatorname{NCSym}_{n}(X)$ is a ring under the basic concatenation product $(K=$ $\{1,2, \ldots, m\} \cup\{m+1, \ldots, m+n\}$. The presence of other shuffle products gives additional structure, which is captured below by the different parabolic subgroups of $U_{n}(q)$.

The ring NCSym naturally generalizes the usual ring of symmetric functions [15], but is different from other generalizations such as the ring of noncommutative symmetric functions studied in, for example, [12]. The ring NCSym was introduced by Wolf [21], and further explored by Rosas and Sagan [17]. There has been recent interest in the Hopf structure of NCSym and its Hopf dual - for example, [7, 8]. In particular, [7] show that it has a representation theoretic connection with partition lattice algebras. This paper suggests that the supercharacter theory of $U_{n}(q)$ also has a representation theoretic connection to NCSym in a way that is more analogous to how the ring of symmetric functions dictates the representation theory of $S_{n}$. However, the precise nature of this connection remains open. In particular, it is not clear whether the Hopf structure of NCSym translates naturally into a representation theoretic Hopf structure for the supercharacters of $U_{n}(q)$. 


\section{The ring of unipotent superclass functions}

This section explores the relationship between NCSym and the space of supercharacters

$$
\mathcal{C}(q)=\bigoplus_{n \geq 0} \mathcal{C}_{n}(q), \quad \text { where } \quad \mathcal{C}_{n}(q)=\mathbb{C}-\operatorname{span}\left\{\chi^{\lambda} \mid \lambda \in \mathcal{S}_{n}(q)\right\}
$$

\subsection{Parabolic subgroups and set-partition combinatorics}

For every $K=K_{1} \cup K_{2} \in \mathcal{S}_{m+n}$ with $\left|K_{1}\right|=m$ and $\left|K_{2}\right|=n, U_{m+n}(q)$ has a parabolic subgroup $U_{m}(q) \times{ }_{K} U_{n}(q)=U_{K}(q) \cong U_{m}(q) \times U_{n}(q)$. However, for different $K$, these isomorphic subgroups are not related via an inner automorphism of $U_{n}(q)$. In fact, it follows from Corollary 4.7, below, that if $U_{K} \cong U_{L} \subseteq U_{n}$ with $K \neq L$, then there exists $\lambda \in \mathcal{S}_{n}(q)$ such that $\operatorname{Res}_{U_{K}}^{U_{n}}\left(\chi^{\lambda}\right) \neq \operatorname{Res}_{U_{L}}^{U_{n}}\left(\chi^{\lambda}\right)$.

This observation gives the space $\mathcal{C}$ a variety of different products. For $\lambda \in \mathcal{S}_{m}(q)$, $\mu \in \mathcal{S}_{n}(q)$, and $K=K_{1} \cup K_{2} \in \mathcal{S}_{m+n}$ with $\left|K_{1}\right|=m$ and $\left|K_{2}\right|=n$, define

$$
\chi^{\lambda} *_{K} \chi^{\mu}=\operatorname{SInd}_{U_{m}(q) \times{ }_{K} U_{n}(q)}^{U_{m+n}(q)}\left(\chi^{\lambda} \times \chi^{\mu}\right) .
$$

There is a related map

$$
\begin{aligned}
\cup_{K}: \mathcal{S}_{m}(q) \times \mathcal{S}_{n}(q) & \longrightarrow \mathcal{S}_{m+n}(q) \\
(\lambda, \mu) & \mapsto \lambda \cup_{K} \mu,
\end{aligned}
$$

where $\lambda \cup_{K} \mu=\lambda^{\prime} \cup \mu^{\prime}$ with $\lambda^{\prime} \in \mathcal{S}_{K_{1}}(q)$ and $\mu^{\prime} \in \mathcal{S}_{K_{2}}(q)$ the same $\mathbb{F}_{q}$-labeled set-partitions as $\lambda$ and $\mu$ respectively, but with $\{1,2, \ldots, m\}$ relabeled as $K_{1}$ and $\{1,2, \ldots, n\}$ relabeled as $K_{2}$. For example,

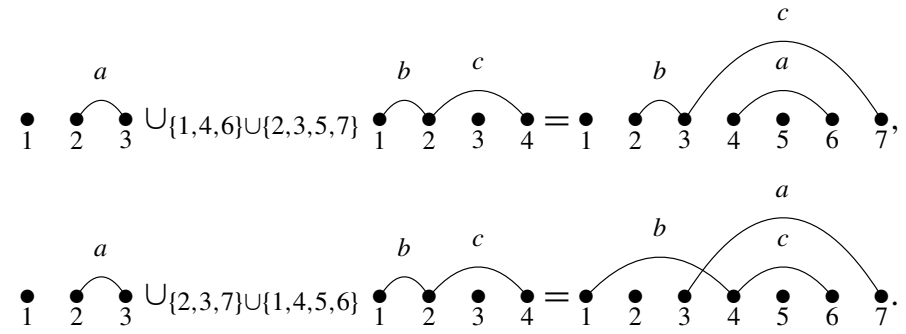

It will follow from Corollary 4.14 that $\chi^{\lambda \cup_{K} \mu}$ is always a nonzero constituent of $\chi^{\lambda} *_{K} \chi^{\mu}$.

Remark The graph automorphism $\sigma$ of the Dynkin diagram of type $A$ gives a natural map

$$
\begin{aligned}
\sigma: \mathcal{C} & \longrightarrow \mathcal{C} \\
\chi^{\lambda} & \mapsto \chi^{\sigma(\lambda)}, \quad \text { for } \lambda \in \mathcal{S}(q),
\end{aligned}
$$

where $\sigma(\lambda) \in \mathcal{S}(q)$ is the $\mathbb{F}_{q}$-labeled set partition obtained by reflecting the diagram $\lambda$ across a vertical axis. This map is an anti-automorphism of $\mathcal{C}$ (it also sends left 
modules to right modules). Thus, in many of the following results we only prove half of the symmetric cases.

3.2 A characteristic map for supercharacters

For $\mu \in \mathcal{S}_{n}(q)$, let $\kappa_{\mu}: U_{n} \rightarrow \mathbb{C}$ be the superclass characteristic function given by

$$
\kappa_{\mu}(u)= \begin{cases}1, & \text { if } u \text { is in the same superclass as } u_{\mu}, \\ 0, & \text { otherwise }\end{cases}
$$

and

$$
z_{\mu}=\frac{\left|U_{n}\right|}{\left|U_{n}\left(u_{\mu}-1\right) U_{n}\right|} .
$$

Proposition 3.1 For $\mu \in \mathcal{S}_{m}(q)$ and $v \in \mathcal{S}_{n}(q)$,

$$
\operatorname{SInd}_{U_{m} \times{ }_{K} U_{n}}^{U_{m+n}}\left(\left(z_{\mu} \kappa_{\mu}\right) \otimes\left(z_{\nu} \kappa_{\nu}\right)\right)=z_{\mu \cup_{K} \nu} \kappa_{\mu \cup_{K} \nu} .
$$

Proof By definition,

$$
\begin{aligned}
& \operatorname{SInd}_{U_{m} \times{ }_{K} U_{n}}^{U_{m+n}}\left(\left(z_{\mu} \kappa_{\mu}\right) \otimes\left(z_{\nu} \kappa_{\nu}\right)\right)(g) \\
& =\frac{z_{\mu} z_{\nu}}{\left|U_{m+n}\right|\left|U_{m}\right|\left|U_{n}\right|} \sum_{\substack{x, y \in U_{m+n} \\
x(g-1) y+1 \in U_{m} \times_{K} U_{n}}}\left(\kappa_{\mu} \otimes \kappa_{\nu}\right)(x(g-1) y+1) \\
& \quad=0,
\end{aligned}
$$

unless the superclass containing $g$ also contains $u_{\mu \cup_{K} v}=u_{\mu} \times_{K} u_{\nu}$. That is, there exists $c \in \mathbb{C}$ such that

$$
\operatorname{SInd}_{U_{m} \times{ }_{K} U_{n}}^{U_{m+n}}\left(\left(z_{\mu} \kappa_{\mu}\right) \otimes\left(z_{\nu} \kappa_{\nu}\right)\right)=c \kappa_{\mu \cup_{K} \nu} .
$$

Specifically,

$$
\begin{aligned}
c & =\frac{z_{\mu} z_{v}}{\left|U_{m+n}\right|\left|U_{m}\right|\left|U_{n}\right|} \sum_{\substack{x, y \in U_{m+n} \\
x(g-1) y+1 \in U_{m} \times X_{K}}}\left(\kappa_{\mu} \otimes \kappa_{v}\right)\left(x\left(u_{\mu \cup_{K} v}-1\right) y+1\right) \\
& =\frac{z_{\mu} z_{v}}{\left|U_{m+n}\right|\left|U_{m}\right|\left|U_{n}\right|}\left|U_{m+n}\right| z_{\mu \cup_{K} v} \sum_{\substack { g-1 \in U_{m+n}\left(u_{\mu \cup_{K} v}-1\right) U_{m+n} \in U_{m} \times U_{n} \\
\begin{subarray}{c}{g \in U_{n}\\
{ g - 1 \in U _ { m + n } ( u _ { \mu \cup _ { K } v } - 1 ) U _ { m + n } \in U _ { m } \times U _ { n } \\
\begin{subarray} { c } { g \in U _ { n } \\
} }\end{subarray}}\left(\kappa_{\mu} \otimes \kappa_{v}\right)(g) \\
& =\frac{z_{\mu} z_{v}}{\left|U_{m+n}\right|\left|U_{m}\right|\left|U_{n}\right|}\left|U_{m+n}\right| z_{\mu \cup_{K} v}\left|U_{m}\left(u_{\mu}-1\right) U_{m}\right|\left|U_{n}\left(u_{v}-1\right) U_{n}\right| \\
& =z_{\mu \cup_{K} v},
\end{aligned}
$$

as desired. 
Let NCSym be the ring of symmetric functions in non-commuting variables. Let

$$
\left\{p_{\lambda} \mid \lambda \in \mathcal{S}\right\}
$$

be any basis that satisfies

$$
p_{\lambda} *_{K} p_{\mu}=p_{\lambda \cup_{K} \mu}
$$

for all $K=K_{1} \cup K_{2} \in \mathcal{S}$ with $\left|K_{1}\right|=|\lambda|$ and $\left|K_{2}\right|=|\mu|$. Note that NCSym in fact has several such bases, such as $\left\{p_{\lambda}\right\}$ in [17] and $\left\{x_{\lambda}\right\}$ in [7].

Corollary 3.2 The function

$$
\begin{aligned}
\operatorname{ch}: \mathcal{C}(2) & \longrightarrow \text { NCSym } \\
\kappa_{\mu} & \mapsto \frac{1}{z_{\mu}} p_{\mu}
\end{aligned}
$$

is an isometric algebra isomorphism.

Questions This result raises the following questions.

(1) Does the Hopf algebra structure of NCSym transfer in a representation theoretic way to $\mathcal{C}$ ?

(2) What is the correct choice of basis $p_{\mu}$ ? In particular, the $\left\{p_{\lambda}\right\}$ of [17] do not seem to give a nice Hopf structure to $\mathcal{C}$.

(3) Is there a corresponding NCSym-space for $q>2$ ?

Questions (1) and (2) presumably need simultaneous answers, and question (3) suggests there might be an analogue of the ring symmetric functions corresponding to wreath products.

\section{Representation theoretic structure constants}

This section explores the computation of structure constants in $\mathcal{C}$. We begin with a family of natural embedding maps of $\mathcal{C}_{m}(q) \subseteq \mathcal{C}_{n}(q)$ for $m \leq n$ using a generalization of the inflation functor, and then give algorithms for computing restrictions from $\mathcal{C}_{m+n}(q)$ to $\mathcal{C}_{m}(q) \otimes \mathcal{C}_{n}(q)$. To finish the computations we require a method for decomposing tensor products $\mathcal{C}_{n}(q) \otimes \mathcal{C}_{n}(q) \rightarrow \mathcal{C}_{n}(q)$. We conclude with a discussion of the corresponding superinduction coefficients. In this section we will assume a fixed $q$, and suppress the $q$ from the notation; that is $U_{n}=U_{n}(q)$, etc.

\subsection{Superinflation of characters}

Let $T \subseteq G$ be pattern groups with corresponding algebras $\mathfrak{t}$ and $\mathfrak{g}$, respectively. Let $\mathfrak{t}^{\perp} \subseteq \mathfrak{g}$ be given by

$$
\mathfrak{t}^{\perp}=\left\{x \in \mathfrak{g} \mid \sum_{i<j} x_{i j} t_{i j}=0, t \in \mathfrak{t}\right\} .
$$


There exists a surjective projection

$$
\begin{aligned}
\pi: \mathfrak{g}=\mathfrak{t} \oplus \mathfrak{t}^{\perp} & \longrightarrow \mathfrak{t} \\
X+Y & \mapsto X,
\end{aligned}
$$

with a corresponding inflation map

$$
\begin{aligned}
\operatorname{Inf}_{\mathfrak{t}}^{\mathfrak{g}}: \mathfrak{t}^{*} & \longrightarrow \mathfrak{g}^{*} \\
\mu & \mapsto \mu \circ \pi .
\end{aligned}
$$

The superinflation map on supermodules is given by

$$
\begin{aligned}
& \operatorname{Sinf}_{T}^{G}:\left\{\begin{array}{c}
\text { Supermodules } \\
\text { of } T
\end{array}\right\} \longrightarrow\left\{\begin{array}{c}
\text { Supermodules } \\
\text { of } G
\end{array}\right\} \\
& V^{\mu} \mapsto V^{\operatorname{Inf}} \mathfrak{t}_{\mathfrak{t}}^{\mathfrak{g}}(\mu)
\end{aligned}
$$

Note that superinflation takes supermodules to supermodules, just as the usual inflation map on characters takes irreducible characters to irreducible characters. Recall, the usual inflation map is constructed from a surjection $\pi: G \rightarrow T$ is given by

$$
\begin{aligned}
\operatorname{Inf}_{T}^{G}:\{T \text {-modules }\} & \longrightarrow\{G \text {-modules }\} \\
V & \mapsto \operatorname{Inf}_{T}^{G}(V),
\end{aligned}
$$

where $g v=\pi(g) v$ for $g \in G, v \in \operatorname{Inf}_{T}^{G}(V)$. The following proposition says that superinflation is inflation whenever possible.

Proposition 4.1 Suppose $G$ is a pattern group with pattern subgroups $T$ and $H$ such that $G=T \ltimes H$. Then for any supermodule $V^{\lambda}$ of $T$,

$$
\operatorname{Sinf}_{T}^{G}\left(V^{\lambda}\right) \cong \operatorname{Inf}_{T}^{G}\left(V^{\lambda}\right)
$$

Proof Let $\mathfrak{g}=G-1, \mathfrak{h}=H-1$, and $\mathfrak{t}=T-1$. Consider the map

$$
\begin{aligned}
\varphi: V^{\operatorname{Inf}_{\mathfrak{t}}^{\mathfrak{g}}(\lambda)} & \longrightarrow \operatorname{Inf}_{T}^{G}\left(V^{\lambda}\right) \\
v_{\mu} & \mapsto v_{\operatorname{Res}_{\mathfrak{t}}^{\mathfrak{g}}(\mu)}
\end{aligned}
$$

Since $T \lambda \subseteq G \lambda$, this map is surjective.

By [16, Lemma 3.2] normality in pattern groups implies "super-normality" in the sense that for $h \in H$ and $g \in G$

$$
g(h-1),(h-1) g \in \mathfrak{h} .
$$

Thus, for $t \in T$ and $h \in H$,

$$
\pi(t h-1)=\pi(t(h-1)+(t-1))=\pi(t(h-1))+\pi(t-1)=t-1,
$$


and similarly $\pi(h t-1)=t-1$. For $s, t \in T$, and $h, k \in H$,

$$
\begin{aligned}
(h t) \operatorname{Inf}_{\mathfrak{t}}^{\mathfrak{g}}(\lambda)(k s-1) & =\operatorname{Inf}_{\mathfrak{t}}^{\mathfrak{g}}(\lambda)\left(t^{-1} h^{-1} k s-t^{-1} h^{-1}\right) \\
& =\operatorname{Inf}_{\mathfrak{t}}^{\mathfrak{g}}(\lambda)\left(t^{-1} s s^{-1} h^{-1} k s-1\right)+\operatorname{Inf}_{\mathfrak{t}}^{\mathfrak{g}}(\lambda)\left(1-t^{-1} h^{-1}\right)
\end{aligned}
$$

and since $s^{-1} h^{-1} k s \in H$,

$$
\begin{aligned}
(h t) \operatorname{Inf}_{\mathfrak{t}}^{\mathfrak{g}}(\lambda)(k s-1) & =\lambda\left(t^{-1} s-1\right)+\lambda\left(1-t^{-1}\right) \\
& =(t \lambda)(s-1) \\
& =t \operatorname{Res}_{\mathfrak{t}}^{\mathfrak{g}}\left(\operatorname{Inf}_{\mathfrak{t}}^{\mathfrak{g}}(\lambda)\right)(s-1) .
\end{aligned}
$$

Since $V^{\operatorname{Inf}} f_{\mathfrak{t}}^{\mathfrak{g}}(\lambda)=\mathbb{C}$-span $\left\{v_{g \operatorname{Inf}_{\mathfrak{t}}^{\mathfrak{g}}(\lambda)} \mid g \in G\right\}$, this computation implies that $\varphi$ is injective.

Finally, for $s, t \in T, h \in H$, and $\mu=s \operatorname{Inf}_{\mathfrak{t}}^{\mathfrak{g}}(\lambda)$,

$$
\begin{aligned}
h t \varphi\left(v_{\mu}\right) & =t v_{\operatorname{Res}_{\mathfrak{t}}^{\mathfrak{g}}(\mu)}=\vartheta\left(\mu\left(t^{-1}-1\right)\right) v_{t \operatorname{Res}_{\mathfrak{t}}^{\mathfrak{g}}(\mu)}=\vartheta\left(\mu\left(t^{-1}-1\right)\right) v_{\operatorname{Res}_{\mathfrak{t}} \mathfrak{g}(h t \mu)} \\
& =\varphi\left(h t v_{\mu}\right),
\end{aligned}
$$

so $\varphi$ is a $G$-module isomorphism.

We will be primarily be interested in the superinflation function between parabolic subgroups of $U_{n}(q)$.

\section{Lemma 4.2}

(a) Let $K, L \in \mathcal{S}$ with $U_{K}(q) \subseteq U_{L}(q)$. For $\lambda \in \mathcal{S}_{K}(q)$,

$$
\operatorname{Sinf}_{U_{K}(q)}^{U_{L}(q)}\left(\chi^{\lambda}\right)=\chi^{\langle\lambda\rangle_{L}} .
$$

(b) Let $K=K_{1} \cup K_{2} \in \mathcal{S}_{m+n}$ with $\left|K_{1}\right|=m$ and $\left|K_{2}\right|=n$. For $\lambda \in S_{m}(q)$ and $\mu \in \mathcal{S}_{n}(q)$,

$$
\operatorname{Sinf}_{U_{m} \times{ }_{K} U_{n}}^{U_{m+n}}\left(\chi^{\lambda} \times \chi^{\mu}\right)=\chi^{\lambda \cup_{K} \mu}
$$

Proof (a) Let $\mathfrak{k}=U_{K}(q)-1$ and $\mathfrak{l}=U_{L}(q)-1$. With the usual identification between $\lambda \in \mathcal{S}(q)$ and the function $\lambda \in \mathfrak{n}^{*}$ given by

$$
\lambda(x)=\sum_{i \stackrel{a}{\curvearrowleft} j \in \mathcal{A}(\lambda)} a x_{i j},
$$

we have

$$
\operatorname{Inf}_{\mathfrak{k}}^{\mathfrak{l}}(\lambda)=\langle\lambda\rangle_{L}
$$

(b) follows from (a). 
For example,

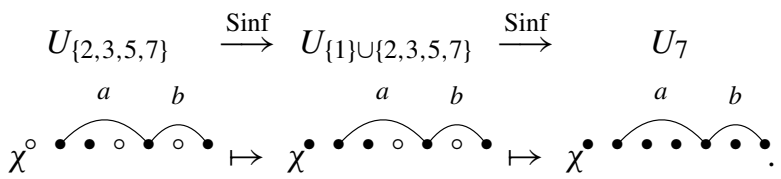

Thus, superinflation allows us to embed $C_{m}(q) \subseteq C_{n}(q)$ for all $m<n$, although this embedding still depends on the embedding of $U_{m}(q)$ inside $U_{n}(q)$.

Remark While the inflation function does match up with the usual inflation when possible, it does not generally behave as nicely as the usual inflation function. In particular, it is no longer generally true that $\operatorname{Res}_{T}^{G} \circ \operatorname{Sinf}_{T}^{G}(\chi)=\chi$ for $\chi$ a class function of $T$. For example,

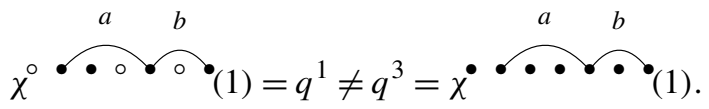

\subsection{Restrictions}

In this section we give algorithms for computing restrictions between parabolic subgroups of $U_{n}(q)$. Since supercharacters decompose into tensor products of arcs, for $\lambda \in \mathcal{S}_{n}(q)$,

$$
\chi^{\lambda}=\prod_{i \stackrel{a}{\lrcorner} l \in \mathcal{A}(\lambda)} \chi^{\langle i \stackrel{a}{-} l\rangle_{n}},
$$

our strategy is to compute restrictions to for each $\chi^{\langle i \stackrel{a}{\sim} l\rangle_{n}}$. We then use a tensor product result in Section 4.3 to glue back together the resulting restrictions.

We begin with two observations, and then Proposition 4.5 and Theorem 4.6 combine to give a general algorithm. Recall that for $K=K_{1} \cup K_{2} \cup \cdots \cup K_{\ell} \in \mathcal{S}_{n}, U_{K}$ is a subgroup of $U_{n}(q)$ isomorphic to

$$
U_{\left|K_{1}\right|} \times U_{\left|K_{2}\right|} \times \cdots \times U_{\left|K_{\ell}\right|}
$$

Proposition 4.3 Let $U_{K} \subseteq U_{L}$ be parabolic subgroups of $U_{n}$ with $L=L_{1} \cup L_{2} \cup$ $\cdots \cup L_{\ell} \in \mathcal{S}_{n}$. Then

$$
\operatorname{Res}_{U_{K}}^{U_{L}}\left(\chi^{\lambda_{1}} \times \cdots \times \chi^{\lambda_{\ell}}\right)=\operatorname{Res}_{U_{K_{1}}}^{U_{L_{1}}}\left(\chi^{\lambda_{1}}\right) \times \operatorname{Res}_{U_{K_{2}}}^{U_{L_{2}}}\left(\chi^{\lambda_{2}}\right) \times \cdots \times \operatorname{Res}_{U_{K_{\ell}}}^{U_{L_{\ell}}}\left(\chi^{\lambda_{\ell}}\right)
$$

where $U_{K_{j}}$ is the parabolic subgroup of $U_{L_{j}}$ corresponding to the vertices $L_{j}$.

The next proposition gives information about each factor in Proposition 4.3. 
Proposition 4.4 For $i<l, a \in \mathbb{F}_{q}^{\times}$and $K=K_{1} \cup K_{2} \cup \ldots \cup K_{\ell} \in \mathcal{S}_{n}$,

$$
\begin{aligned}
\operatorname{Res}_{U_{K}}^{U_{n}}\left(\chi^{\langle i \stackrel{a}{\sim} l\rangle_{n}}\right)= & \frac{\operatorname{Res}_{U_{K_{1}}}^{U_{n}}\left(\chi^{\langle i \stackrel{a}{\sim} l\rangle_{n}}\right)}{q^{\left|\left\{i<k<l \mid k \notin K_{1}\right\}\right|}} \times \frac{\operatorname{Res}_{U_{K_{2}}}^{U_{n}}\left(\chi^{\langle i \stackrel{a}{\sim} l\rangle_{n}}\right)}{q^{\left|\left\{i<k<l \mid k \notin K_{2}\right\}\right|}} \times \cdots \\
& \times \frac{\operatorname{Res}_{U_{K_{\ell}}}^{U_{n}}\left(\chi^{\langle i \stackrel{a}{l} l\rangle_{n}}\right)}{q^{\left|\left\{i<k<l \mid k \notin K_{\ell}\right\}\right|}} .
\end{aligned}
$$

Proof It follows from (2.1) (see also [19] for a more general result) that we can factor the character values across the direct product as

$$
\begin{aligned}
& \operatorname{Res}_{U_{K}}^{U_{n}}\left(\chi^{\langle i \stackrel{a}{\curvearrowleft} l\rangle_{n}}\right)\left(u_{\mu^{(1)}}, \ldots, u_{\mu^{(\ell)}}\right)=\chi^{\langle i \stackrel{a}{ } l\rangle_{n}}(1) \prod_{j=1}^{\ell} \frac{\chi^{\langle i \stackrel{a}{\curvearrowleft} l\rangle_{n}}\left(u_{\mu^{(j)}}\right)}{\chi^{\langle i \stackrel{a}{\curvearrowleft} l\rangle_{n}}(1)} \\
& =\chi^{\langle i \stackrel{a}{\sim} l\rangle_{n}}(1) \prod_{j=1}^{\ell} \frac{\operatorname{Res}_{U_{K_{j}}}^{U_{n}}\left(\chi^{\langle i \stackrel{a}{\sim} l\rangle_{n}}\right)\left(u_{\mu}(j)\right)}{\operatorname{Res}_{U_{K_{j}}}^{U_{n}}\left(\chi^{\langle i \stackrel{a}{\curvearrowleft} l\rangle_{n}}\right)(1)} \text {, }
\end{aligned}
$$

and by (2.2),

$$
\begin{aligned}
& =q^{l-i-1} \prod_{j=1}^{\ell} \frac{\operatorname{Res}_{U_{K_{j}}}^{U_{n}}\left(\chi^{\langle i \stackrel{a}{\sim} l\rangle_{n}}\right)\left(u_{\mu^{(j)}}\right)}{q^{l-i-1}} \\
& =\frac{1}{q^{(\ell-1)(l-i-1)}} \prod_{j=1}^{\ell} \operatorname{Res}_{U_{K_{j}}}^{U_{n}}\left(\chi^{\langle i \stackrel{a}{ } l\rangle_{n}}\right)\left(u_{\mu^{(j)}}\right) \\
& =\prod_{j=1}^{\ell} \frac{\operatorname{Res}_{U_{K_{j}}}^{U_{n}}\left(\chi^{\langle i \stackrel{a}{\curvearrowleft} l\rangle_{n}}\right)\left(u_{\mu^{(j)}}\right)}{q^{\left|\left\{i<k<l \mid k \notin K_{j}\right\}\right|}},
\end{aligned}
$$

as desired.

To compute restrictions, we first consider very specific subgroups of $U_{n}(q)$. For $1<j<k$, let

$$
[j, k]=\{j, j+1, \ldots, k-1, k\}
$$

and

$$
U_{[i, l]}=\left\{u \in U_{n} \mid u_{j k} \neq 0 \text { implies } i \leq j \leq k \leq l\right\} .
$$


Proposition 4.5 Let $1 \leq j<k \leq n$ and $A=[j, k]$. For $1 \leq i<l \leq n$ and $a \in \mathbb{F}_{q}^{\times}$,

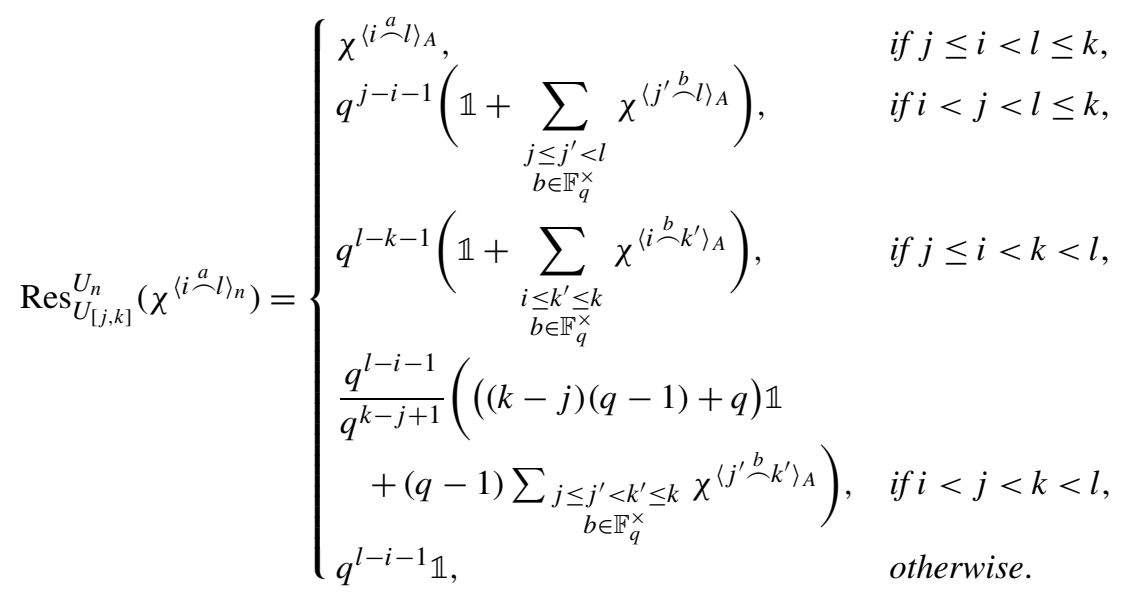

Proof Cases 1 and 5 follow directly from (2.1).

For Case 2, first assume that $i=1, j=2$ and $l \leq k=n$. Then [20] implies the result $\left(q^{j-i-1}=1\right.$ in this special case).

To obtain the remaining cases we iterate by removing one column or row at a time. For $i<j<l \leq k \leq n$,

$$
\operatorname{Res}_{U_{[j, k]}}^{U_{n}}\left(\chi^{\langle i \stackrel{a}{\sim} l\rangle_{n}}\right)=\operatorname{Res}_{U_{[j, k]}}^{U_{[j, n]}} \operatorname{Res}_{U_{[j, n]}}^{U_{n}}\left(\chi^{\langle i \stackrel{a}{\sim} l\rangle_{n}}\right),
$$

where

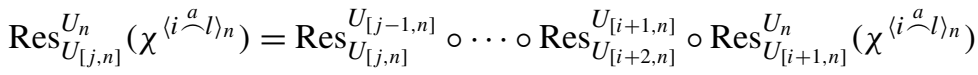

$$
\begin{aligned}
& =\operatorname{Res}_{U_{[j, n]}}^{U_{[j-1, n]}} \circ \cdots \circ \operatorname{Res}_{U_{[i+2, n]}}^{U_{[i+1, n]}}\left(\mathbb{1}+\sum_{\substack{i+1 \leq j^{\prime}<l \\
b \in \mathbb{F}_{q}^{\times}}} \chi^{\left\langle j^{\prime} \stackrel{b}{-} l\right\rangle_{[i+1, n]}}\right) \\
& =\operatorname{Res}_{U_{[j, n]}}^{U_{[j-1, n]}} \circ \cdots \circ \operatorname{Res}_{U_{[i+3, n]}}^{U_{[i+2, n]}}\left(\mathbb{1}+\sum_{\substack{i+2 \leq j^{\prime}<l \\
b \in \mathbb{F}_{q}^{\times}}} \chi^{\left\langle j^{\prime} \stackrel{b}{h} l\right\rangle_{[i+2, n]}}\right.
\end{aligned}
$$

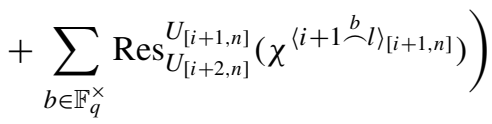

$$
\begin{aligned}
& =\operatorname{Res}_{U_{[j, n]}}^{U_{[j-1, n]}} \circ \cdots \circ \operatorname{Res}_{U_{[i+3, n]}}^{U_{[i+2, n]}}\left(q \mathbb{1}+q \sum_{\substack{i+2 \leq j^{\prime}<l \\
b \in \mathbb{F}_{q}^{\times}}} \chi^{\left\langle j^{\prime} \stackrel{b}{-} l\right\rangle_{[i+2, n]}}\right) \\
& =q \operatorname{Res}_{U_{[j, n]}}^{U_{[j-1, n]}} \circ \cdots \circ \operatorname{Res}_{U_{[i+3, n]}}^{U_{[i+2, n]}}\left(\mathbb{1}+\sum_{\substack{i+2 \leq j^{\prime}<l \\
b \in \mathbb{F}_{q}^{\times}}} \chi^{\left\langle j^{\prime} \stackrel{b}{-} l\right\rangle_{[i+2, n]}}\right)
\end{aligned}
$$


and iterate to obtain

$$
=q^{j-i-1} \mathbb{1}+q^{j-i-1} \sum_{\substack{j \leq j^{\prime}<l \\ b \in \mathbb{F}_{q}^{\times}}} \chi^{\left\langle j^{\prime} \stackrel{b}{ } l^{\circ}\right\rangle_{A}},
$$

giving Case 2. Case 3 follows by a symmetric argument.

If $i<j<k<l$, then by Case 2 ,

$$
\begin{aligned}
& \operatorname{Res}_{U_{[j, k]}}^{U_{n}}\left(\chi^{\langle i \stackrel{a}{\sim} l\rangle_{n}}\right)=\operatorname{Res}_{U_{[j, k]}}^{U_{[j, n]}} \circ \operatorname{Res}_{U_{[j, n]}}^{U_{n}}\left(\chi^{\langle i \stackrel{a}{\sim} l\rangle_{n}}\right) \\
& =\operatorname{Res}_{U_{[j, k]}}^{U_{[j, n]}}\left(q^{j-i-1} \mathbb{1}+q^{j-i-1} \sum_{\substack{j \leq j^{\prime}<l \\
b \in \mathbb{F}_{q}^{\times}}} \chi^{\left\langle j^{\prime} \stackrel{b}{\sim} l\right\rangle_{[j, n]}}\right) \\
& =\operatorname{Res}_{U_{[j, k]}}^{U_{[j, n]}}\left(q^{j-i-1} \mathbb{1}+q^{j-i-1} \sum_{\substack{j \leq j^{\prime}<k \\
b \in \mathbb{F}_{q}^{\times}}} \chi^{\left\langle j^{\prime} \stackrel{b}{\sim} l\right\rangle_{[j, n]}}+q^{j-i-1} \sum_{\substack{k \leq j^{\prime}<l \\
b \in \mathbb{F}_{q}^{\times}}} \chi^{\left\langle j^{\prime} \stackrel{b}{-} l\right\rangle_{[j, n]}}\right),
\end{aligned}
$$

and by Case 3,

$$
\begin{aligned}
& =q^{j-i-1} \mathbb{1}+q^{j-i-1} \sum_{\substack{j \leq j^{\prime}<k \\
b \in \mathbb{F}_{q}^{\times}}}\left(q^{l-k-1} \mathbb{1}+q^{l-k-1} \sum_{\substack{j^{\prime}<k^{\prime} \leq k \\
c \in \mathbb{F}_{q}^{\times}}} \chi^{\left\langle j^{\prime} \stackrel{c}{\triangle} k^{\prime}\right\rangle_{A}}\right) \\
& +q^{j-i-1}(q-1) \sum_{k \leq j^{\prime}<l} q^{l-j^{\prime}-1} \mathbb{1} \\
& =q^{j-i-1} \mathbb{1}+q^{l-k+j-i-2}(k-j)(q-1) \mathbb{1}+q^{l-k+j-i-2} \sum_{\substack{j \leq j^{\prime}<k^{\prime} \leq k \\
b, c \in \mathbb{F}_{q}^{\times}}} \chi^{\left\langle j^{\prime} \stackrel{c}{\frown} k^{\prime}\right\rangle_{A}} \\
& +q^{j-i-1}\left(q^{l-k}-1\right) \mathbb{1} \\
& =\left(q^{l-k+j-i-2}(q-1)(k-j)+q^{l-k+j-i-1}\right) \mathbb{1} \\
& +q^{l-k+j-i-2}(q-1) \sum_{\substack{j \leq j^{\prime}<k^{\prime} \leq k \\
c \in \mathbb{F}_{q}^{\times}}} \chi^{\left\langle j^{\prime} \stackrel{c}{-} k^{\prime}\right\rangle_{A}} \\
& =\frac{q^{l-i-1}}{q^{k-j+1}}\left(((k-j)(q-1)+q) \mathbb{1}+(q-1) \sum_{\substack{j \leq j^{\prime}<k^{\prime} \leq k \\
c \in \mathbb{F}_{q}^{\times}}} \chi^{\left\langle j^{\prime} \stackrel{c}{\sim} k^{\prime}\right\rangle_{A}}\right),
\end{aligned}
$$

giving Case 4. 
Example Let $n=7, j=2, k=5$, so that

$$
U_{[2,5]}=\left\{\left(\begin{array}{lllllll}
1 & 0 & 0 & 0 & 0 & 0 & 0 \\
0 & 1 & * & * & * & 0 & 0 \\
0 & 0 & 1 & * & * & 0 & 0 \\
0 & 0 & 0 & 1 & * & 0 & 0 \\
0 & 0 & 0 & 0 & 1 & 0 & 0 \\
0 & 0 & 0 & 0 & 0 & 1 & 0 \\
0 & 0 & 0 & 0 & 0 & 0 & 1
\end{array}\right)\right\} \subseteq\left\{\left(\begin{array}{lllllll}
1 & * & * & * & * & * & * \\
0 & 1 & * & * & * & * & * \\
0 & 0 & 1 & * & * & * & * \\
0 & 0 & 0 & 1 & * & * & * \\
0 & 0 & 0 & 0 & 1 & * & * \\
0 & 0 & 0 & 0 & 0 & 1 & * \\
0 & 0 & 0 & 0 & 0 & 0 & 1
\end{array}\right)\right\}=U_{7}
$$

Then

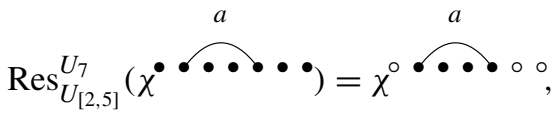

$$
\begin{aligned}
& \operatorname{Res}_{U_{[2,5]}}^{U_{7}}\left(\chi^{a \cdots \cdots \cdots}\right)=\chi^{\circ \cdots \cdots \circ \circ}+\sum_{b \in \mathbb{F}_{q}^{\times}} \chi^{\circ \cdots \bullet \circ \circ}+\sum_{b \in \mathbb{F}_{q}^{\times}} \chi^{\circ} \cdots \bullet \circ \\
& +\sum_{b \in \mathbb{F}_{q}^{\times}} \chi^{\circ \cdots \curvearrowright \circ \circ} \\
& \operatorname{Res}_{U_{[2,5]}^{U_{7}}}\left(\chi^{a} \bullet \bullet \bullet\right)=q\left((4 q-3) \chi^{\circ \cdots \cdots \circ \circ}+(q-1) \sum_{b \in \mathbb{F}_{q}^{\times}} \chi^{\circ} \stackrel{b}{b} \circ \circ\right. \\
& +(q-1) \sum_{b \in \mathbb{F}_{q}^{\times}} \chi^{\circ \cdots \bullet \circ \circ} \\
& +(q-1) \sum_{b \in \mathbb{F}_{q}^{\times}} \chi^{\circ \cdots \curvearrowright \circ \circ}+(q-1) \sum_{b \in \mathbb{F}_{q}^{\times}} \chi^{\circ} \cdots \circ \circ \\
& +(q-1) \sum_{b \in \mathbb{F}_{q}^{\times}} \chi^{\circ \cdots \bullet \circ \circ} \\
& \left.+(q-1) \sum_{b \in \mathbb{F}_{q}^{\times}} \chi^{\circ} \stackrel{b}{\circ} \cdot \circ \circ\right)
\end{aligned}
$$




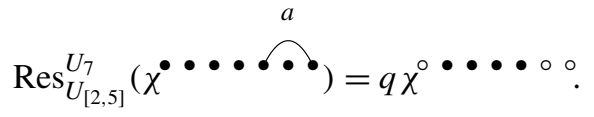

Theorem 4.6, below, generalizes Proposition 4.5 to the required level of generality. For $A \subseteq\{1,2, \ldots, n\}$, let

$$
U_{A}=\left\{u \in U_{n} \mid u_{i j} \neq \text { 0implies } i, j \in A\right\} .
$$

Note that while $U_{A}$ is not itself a parabolic subgroup of $U_{n}$, it is isomorphic to the parabolic subgroup $U_{\langle A\rangle_{n}}$.

Theorem 4.6 Let $A \subseteq\{1,2, \ldots, n\}$. Then for $1 \leq i<l \leq n$ and $a \in \mathbb{F}_{q}^{\times}$,

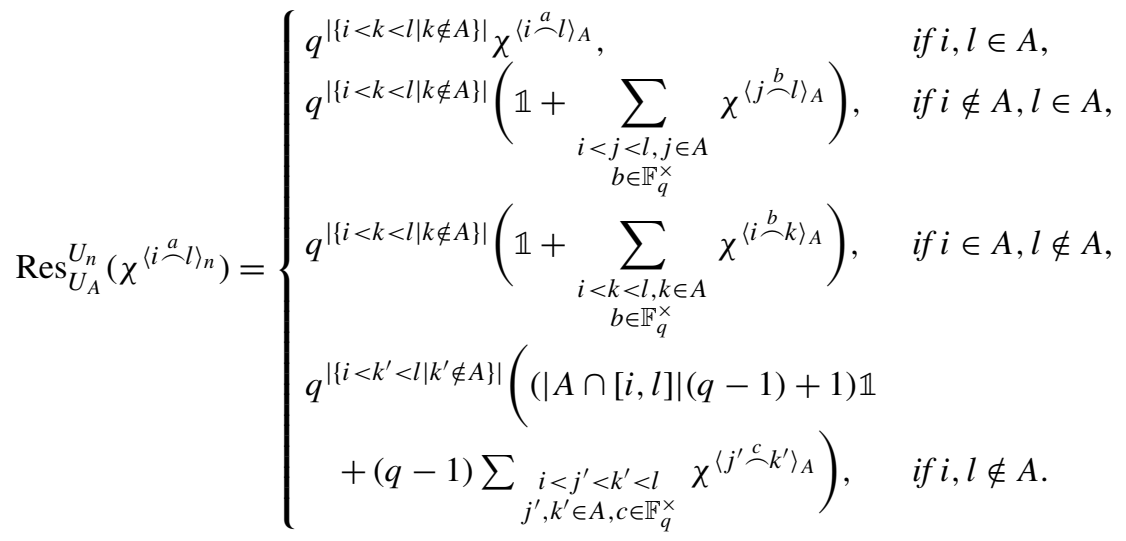

Proof Note that it follows from the character formulas (2.1) and (2.2) that if $i, l \in A$, then

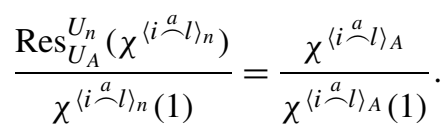

Therefore,

$$
\operatorname{Res}_{U_{A}}^{U_{n}}\left(\chi^{\langle i \stackrel{a}{\neg} l\rangle_{n}}\right)=\frac{\chi^{\langle i \stackrel{a}{\curvearrowleft} l\rangle_{n}}(1)}{\chi^{\langle i \stackrel{a}{\curvearrowleft} l\rangle_{A}(1)}} \chi^{\langle i \stackrel{a}{\curvearrowleft} l\rangle_{A}}=q^{|\{i<k<l \mid k \notin A\}|} \chi^{\langle i \stackrel{a}{ } l\rangle_{A}}
$$

gives the first case.

For Case 2, assume that $i \notin A$, and $l \in A$. By Proposition 4.5, Case 2 and then (4.1),

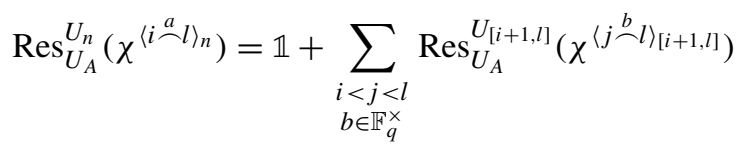




$$
\begin{gathered}
=\mathbb{1}+\sum_{\substack{i<j<l, j \in A \\
b \in \mathbb{F}_{q}^{\times}}} q^{|\{j<k<l \mid k \notin A\}|} \chi^{\langle j \stackrel{b}{\circ} l\rangle_{A}} \\
+\sum_{\substack{i<j<l, j \notin A \\
b \in \mathbb{F}_{q}^{\times}}} \operatorname{Res}_{U_{A}}^{U_{[i+1, l]}}\left(\chi^{\langle j \stackrel{b}{-} l\rangle_{[i+1, l]}}\right) .
\end{gathered}
$$

If $j^{\prime}$ is minimal such that $i<j^{\prime}<l$ and $j^{\prime} \notin A$, then by Proposition 4.5, Case 2,

$$
\begin{aligned}
& \sum_{\substack{i<j<l, j \notin A \\
b \in \mathbb{F}_{q}^{\times}}} \operatorname{Res}_{U_{A}}^{U_{[i+1, l]}}\left(\chi^{\langle j \stackrel{b}{\sim} l\rangle_{[i+1, l]}}\right) \\
& =\sum_{b \in \mathbb{F}_{q}^{\times}} \operatorname{Res}_{U_{A}}^{U_{\left[j^{\prime}+1, l\right]}} \operatorname{Res}_{U_{\left[j^{\prime}+1, l\right]}}^{U_{[i+1, l]}}\left(\chi^{\left\langle j^{\prime} \stackrel{b}{-} l\right\rangle_{[i+1, l]}}\right) \\
& +\sum_{\substack{j^{\prime}<j<l, j \notin A \\
b \in \mathbb{F}_{q}^{\times}}} \operatorname{Res}_{U_{A}}^{U_{\left[j^{\prime}+1, l\right]}}\left(\chi^{\langle j \stackrel{b}{-} l\rangle_{\left[j^{\prime}+1, l\right]}}\right) \\
& =(q-1) \mathbb{1}+(q-1) \sum_{\substack{j^{\prime}<j<l \\
c \in \mathbb{F}_{q}^{\times}}} \operatorname{Res}_{U_{A}}^{U_{\left[j^{\prime}+1, l\right]}}\left(\chi^{\left\langle j \stackrel{c}{ }{ }^{c} l\right\rangle_{\left[j^{\prime}+1, l\right]}}\right)
\end{aligned}
$$

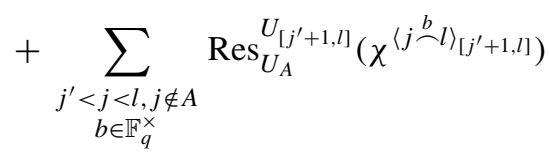

$$
\begin{aligned}
& =(q-1) \mathbb{1}+(q-1) \sum_{\substack{j^{\prime}<j<l, j \in A \\
c \in \mathbb{F}_{q}^{\times}}} q^{|\{j<k<l \mid k \notin A\}|} \chi^{\langle j \stackrel{c}{\sim} l\rangle_{A}} \\
& +q \sum_{\substack{j^{\prime}<j<l, j \notin A \\
c \in \mathbb{F}_{q}^{\times}}} \operatorname{Res}_{U_{A}}^{U_{\left[j^{\prime}+1, l\right]}}\left(\chi^{\langle j \stackrel{c}{ }-l\rangle_{\left[j^{\prime}+1, l\right]}}\right) .
\end{aligned}
$$

Applying this equality to (4.2),

$$
\begin{aligned}
\operatorname{Res}_{U_{A}}^{U_{n}}\left(\chi^{\langle i \stackrel{a}{\sim} l\rangle_{n}}\right)= & q \mathbb{1}+q^{|\{i<k<l \mid k \notin A\}|} \sum_{\substack{i<j<j^{\prime} \\
b \in \mathbb{F}_{q}^{\times}}} \chi^{\langle j \stackrel{b}{\sim} l\rangle_{A}} \\
& +q \sum_{\substack{j^{\prime}<j<l, j \in A \\
b \in \mathbb{F}_{q}^{\times}}} q^{|\{j<k<l \mid k \notin A\}|} \chi^{\langle j \stackrel{b}{-} l\rangle_{A}}
\end{aligned}
$$




$$
\begin{aligned}
& +q \sum_{\substack{j^{\prime}<j<l, j \notin A \\
c \in \mathbb{F}_{q}^{\times}}} \operatorname{Res}_{U_{A}}^{U_{\left[j^{\prime}+1, l\right]}}\left(\chi^{\langle j \stackrel{c}{-} l\rangle_{\left[j^{\prime}+1, l\right]}}\right) \\
& =q \mathbb{1}+q^{|\{i<k<l \mid k \notin A\}|} \sum_{\substack{i<j<j^{\prime \prime} \\
b \in \mathbb{F}_{q}^{\times}}} \chi^{\langle j \stackrel{b}{ }-l\rangle_{A}} \\
& +q\left(\sum_{\substack{j^{\prime \prime}<j<l, j \in A \\
b \in \mathbb{F}_{q}^{\times}}} q^{|\{j<k<l \mid k \notin A\}|} \chi^{\langle j \stackrel{b}{-} l\rangle_{A}}\right.
\end{aligned}
$$

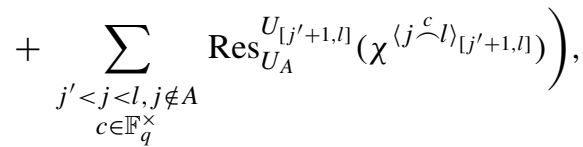

where $j^{\prime \prime}$ is minimal such that $j^{\prime}<j^{\prime \prime}<l$ and $j^{\prime \prime} \notin A$. Iterating this process gives Case 2. A symmetric argument also gives Case 3 .

Suppose $i, l \notin A$ with $|A \cap[i, l]| \neq 0$. Let $k^{\prime}<l$ maximal such that $k^{\prime} \in A$. We apply Cases 2 and 3 consecutively, to deduce Case 3 . By Case 2,

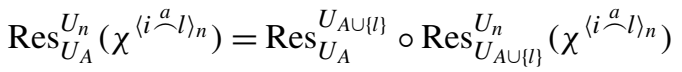

$$
\begin{aligned}
& =q^{|\{i<k<l \mid k \notin A\}|} \operatorname{Res}_{U_{A}}^{U_{A \cup\{l\}}}\left(\mathbb{1}+\sum_{\substack{i<j<l, j \in A \\
b \in \mathbb{F}_{q}^{\times}}} \chi^{\langle j \stackrel{b}{-} l\rangle_{A \cup\{l\}}}\right) \\
& =q^{|\{i<k<l \mid k \notin A\}|}\left(\mathbb{1}+\sum_{\substack{i<j<l, j \in A \\
b \in \mathbb{F}_{q}^{\times}}} \operatorname{Res}_{U_{A}}^{U_{A \cup\{l\}}}\left(\chi^{\langle j \stackrel{b}{-} l\rangle_{A \cup\{l\}}}\right)\right) \\
& =q^{|\{i<k<l \mid k \notin A\}|}\left(\mathbb{1}+\sum_{\substack{i<j<k^{\prime}, j \in A \\
b \in \mathbb{F}_{q}^{\times}}} \operatorname{Res}_{U_{A}}^{U_{A \cup\{l\}}}\left(\chi^{\left.\langle j \stackrel{b}{-} l\rangle_{A \cup\{l\}}\right)}\right.\right.
\end{aligned}
$$

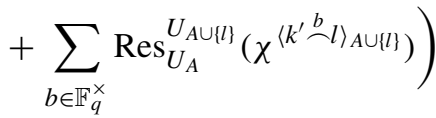

and then by Case 3,

$$
\begin{aligned}
& =q^{|\{i<k<l \mid k \notin A\}|}\left(\mathbb{1}+\left|A \cap\left[i, k^{\prime}\right)\right|(q-1) \mathbb{1}\right. \\
& +(q-1) \sum_{\substack{i<j<k<l, j, k \in A \\
c \in \mathbb{F}_{q}^{\times}}} \chi^{\left\langle j{\stackrel{c}{c} k\rangle_{A}}^{\times}+(q-1) \mathbb{1}\right)}
\end{aligned}
$$




$$
\begin{aligned}
& =q^{|\{i<k<l \mid k \notin A\}|}\left((|A \cap[i, k)|(q-1)+q) \mathbb{1}+(q-1) \sum_{\substack{i<j<k<l, j, k \in A \\
c \in \mathbb{F}_{q}^{\times}}} \chi^{\langle j \stackrel{c}{c} k\rangle_{A}}\right) \\
& =q^{|\{i<k<l \mid k \notin A\}|}(((|A \cap[i, l]|-1)(q-1)+q) \mathbb{1} \\
& \left.+(q-1) \sum_{\substack{i<j<k<l, j, k \in A \\
c \in \mathbb{F}_{q}^{\times}}} \chi^{\left\langle j \stackrel{c}{c}^{\times} k\right\rangle_{A}}\right) \\
& =q^{|\{i<k<l \mid k \notin A\}|}\left((|A \cap[i, l]|(q-1)+1) \mathbb{1}+(q-1) \sum_{\substack{i<j<k<l, j, k \in A \\
c \in \mathbb{F}_{q}^{\times}}} \chi^{\left\langle j{ }^{c} k\right\rangle_{A}}\right) .
\end{aligned}
$$

On the other hand, if $i, l \notin A$ and $|A \cap[i, l]|=0$, then there exists $j<k$ such that $A \subseteq[j, k]$ and $[i, l] \cap[j, k]=\emptyset$. Thus, by Proposition 4.5, Case 5,

$$
\begin{aligned}
\operatorname{Res}_{U_{A}}^{U_{n}}\left(\chi^{\langle i \stackrel{a}{\neg} l\rangle_{n}}\right)= & \operatorname{Res}_{U_{A}}^{U_{[j, k]}} \circ \operatorname{Res}_{U_{[j, k]}}^{U_{n}}\left(\chi^{\langle i \stackrel{a}{\neg} l\rangle_{n}}\right) \\
= & q^{l-i-1} \mathbb{1} \\
= & q^{|\{i<k<l \mid k \notin A\}|}((|A \cap[i, l]|(q-1)+1) \mathbb{1} \\
& \left.+(q-1) \sum_{\substack{i<j<k<l, j, k \in A \\
c \in \mathbb{F}_{q}^{\times}}} \chi^{\left\langle j \stackrel{c}{c}^{c} k\right\rangle_{A}}\right),
\end{aligned}
$$

where the sum is empty in this case.

Corollary 4.7 If $A, B \subseteq\{1,2, \ldots, n\}$ with $U_{A} \cong U_{B} \cong U$ and $A \neq B$, then there exists $\lambda \in \mathcal{S}_{n}(q)$ such that $\operatorname{Res}_{U_{A}}^{U_{n}}\left(\chi^{\lambda}\right)$ and $\operatorname{Res}_{U_{B}}^{U_{n}}\left(\chi^{\lambda}\right)$ are different characters of $U$.

Proof We may assume $A \neq \emptyset$. Else, $U_{A} \cong U_{B}$ implies $A=B$. If $A \cap B=\emptyset$, then for $\mathcal{A}(\lambda)=\{i \stackrel{1}{\sim} k\}$ with $i, k \in A$,

$$
\begin{aligned}
\operatorname{Res}_{U_{A}}^{U_{n}}\left(\chi^{\lambda}\right)= & q^{\mid\{i<j<k \mid j \notin A\}} \chi^{\langle i \stackrel{1}{1} k\rangle_{A}} \\
\operatorname{Res}_{U_{B}}^{U_{n}}\left(\chi^{\lambda}\right)= & q^{|\{i<j<k \mid j \notin B\}|}((|B \cap[i, k]|(q-1)+1) \mathbb{1} \\
& \left.+(q-1) \sum_{\substack{i<j<k^{\prime}<k \\
j, k^{\prime} \in B, c \in \mathbb{F}_{q}^{\times}}} \chi^{\left\langle j \stackrel{c}{\complement} k^{\prime}\right\rangle_{B}}\right) .
\end{aligned}
$$

Thus, $\operatorname{Res}_{U_{A}}^{U_{n}}\left(\chi^{\lambda}\right)$ is a multiple of a nontrivial supercharacter, whereas $\operatorname{Res}_{U_{B}}^{U_{n}}\left(\chi^{\lambda}\right)$ is 
either trivial (up to a scalar multiple) or a linear combination of at least two different supercharacters.

If $A \cap B \neq \emptyset$, then for $\mathcal{A}(\lambda)=\{i \stackrel{1}{\sim} k\}$ with $i \in A \cap B, k \in A-B$ (WLOG $i<k$ ),

$$
\begin{aligned}
& \operatorname{Res}_{U_{A}}^{U_{n}}\left(\chi^{\lambda}\right)=q^{\mid\{i<j<k \mid j \notin A\}} \chi^{\langle i \stackrel{1}{\sim} k\rangle_{A}} \\
& \operatorname{Res}_{U_{B}}^{U_{n}}\left(\chi^{\lambda}\right)=q^{|\{i<j<k \mid j \notin B\}|}\left(\mathbb{1}+\sum_{\substack{i<j<k \\
j \in B, c \in \mathbb{F}_{q}^{\times}}} \chi^{\langle i \stackrel{c}{\sim} j\rangle_{B}}\right) .
\end{aligned}
$$

Thus, $\operatorname{Res}_{U_{A}}^{U_{n}}\left(\chi^{\lambda}\right)$ is a multiple of a nontrivial supercharacter, whereas $\operatorname{Res}_{U_{B}}^{U_{n}}\left(\chi^{\lambda}\right)$ is either trivial (up to a scalar multiple) or a linear combination of at least two different supercharacters.

\subsection{Tensor products}

We have seen in the previous section that when we decompose supercharacters into tensor products of irreducible characters, the restriction rules are manageable to compute. This section explains how to glue back together the resulting products of characters. The main result - Corollary 4.9 - has been computed by André for large primes in [1, Lemmas 6-8] and for arbitrary primes by Yan in [22, Propositions 7.2-7.5], but we reprove it here quickly using the machinery developed in this paper.

We begin with a lemma that further establishes the relationship between tensor products and restrictions. For $H \subseteq G$ and $\chi$ a superclass function of $G$, let

$$
\operatorname{SinfRes}_{H}^{G}(\chi)=\operatorname{Sinf}_{H}^{G} \operatorname{Res}_{H}^{G}(\chi) .
$$

Lemma 4.8 For $i<j<k<l$,

$$
\begin{aligned}
& \chi^{\langle i \stackrel{a}{\sim} k\rangle_{n}} \otimes \chi^{\langle i \stackrel{b}{\sim} l\rangle_{n}}=\operatorname{SinfRes}_{U_{[i+1, l]}}^{U_{n}}\left(\chi^{\langle i \stackrel{a}{\sim} k\rangle_{n}}\right) \otimes \chi^{\langle i \stackrel{b}{\sim} l\rangle_{n}}, \quad a, b \in \mathbb{F}_{q}^{\times}, \\
& \chi^{\langle i \stackrel{a}{\sim} l\rangle_{n}} \otimes \chi^{\langle j \stackrel{b}{\sim} l\rangle_{n}}=\chi^{\langle i \stackrel{a}{\sim} l\rangle_{n}} \otimes \operatorname{SinfRes}_{U_{[i, l-1]}}^{U_{n}}\left(\chi^{\langle j \stackrel{b}{ } l\rangle_{n}}\right), \quad a, b \in \mathbb{F}_{q}^{\times}, \\
& \chi^{\langle i \stackrel{a}{\neg} l\rangle_{n}} \otimes \chi^{\left\langle i i^{-a} l\right\rangle_{n}}=\operatorname{SinfRes}_{U_{[i+1, l]}}^{U_{n}}\left(\chi^{\langle i \stackrel{a}{\sim} l\rangle_{n}}\right) \otimes \operatorname{SinfRes}_{U_{[i, l-1]}}^{U_{n}}\left(\chi^{\langle i \stackrel{-a}{\sim} l\rangle_{n}}\right), \quad a \in \mathbb{F}_{q}^{\times}, \\
& \chi^{\langle i \stackrel{a}{\sim} l\rangle_{n}} \otimes \chi^{\langle i \stackrel{b}{\sim} l\rangle_{n}}=\chi^{\left\langle i i^{a+b} \sim\right\rangle_{n}} \otimes \operatorname{SinfRes}_{U_{[i+1, l-1]}}^{U_{n}}\left(\chi^{\langle i \stackrel{a+b}{\sim} l\rangle_{n}}\right), \quad a, b \in \mathbb{F}_{q}^{\times}, b \neq-a .
\end{aligned}
$$

Proof For the first case, note that by (2.1),

$$
\begin{aligned}
& \chi^{\langle i \stackrel{a}{\sim} k\rangle_{n}} \otimes \chi^{\langle i \stackrel{b}{ } l\rangle_{n}}\left(u_{\mu}\right) \\
& = \begin{cases}\frac{\chi^{\langle i \stackrel{a}{\neg} k\rangle_{n}}(1) \chi^{\langle i \stackrel{b}{\neg} l\rangle_{n}}(1) \vartheta\left(b \tau_{\mu}(i \frown l)\right)}{q^{\left|\left\{j^{\prime} \frown k^{\prime} \in \mu \mid i<j^{\prime}<k^{\prime}<k\right\}\right|+\left|\left\{j^{\prime} \frown k^{\prime} \in \mu \mid i<j^{\prime}<k^{\prime}<l\right\}\right|},} & i \frown j^{\prime}, j^{\prime} \frown k \notin \mathcal{A}(\mu), \\
0, & i<j^{\prime}<l \text { implies } \\
0, & i \frown j^{\prime}, j^{\prime} \frown l \notin \mathcal{A}(\mu) ; \\
\text { otherwise, }\end{cases}
\end{aligned}
$$


where the $\vartheta\left(a \tau_{\mu}(i \frown k)\right)$ term is missing since if $i \frown k \in \mathcal{A}(\mu)$ then the character value is zero. Now the first case follows from the observation that for $v \in \mathcal{S}_{[i+1, l]}(q)$,

$$
\operatorname{Res}_{U_{[i+1, l]}}^{U_{n}}\left(\chi^{\langle i \stackrel{a}{\sim} k\rangle_{n}}\right)\left(u_{v}\right)=\left\{\begin{array}{l}
\frac{\chi^{\langle i \stackrel{a}{\sim} k\rangle_{n}}(1)}{q^{\left|\left\{j^{\prime} \frown k^{\prime} \in v \mid i<j^{\prime}<k^{\prime}<k\right\}\right|},} \\
\text { if } i<j^{\prime}<k \text { implies } j^{\prime} \frown k \notin A(v), \\
0, \quad \text { otherwise. }
\end{array}\right.
$$

Case 2 follows from a symmetric argument, and Cases 3 and 4 are proved by a similar argument.

Combining Lemma 4.9 with Proposition 4.5 we obtain the following corollary.

Corollary 4.9 For $i<k, j<l, a, b \in \mathbb{F}_{q}^{\times}$, and $\{i, k\} \neq\{j, l\}$,

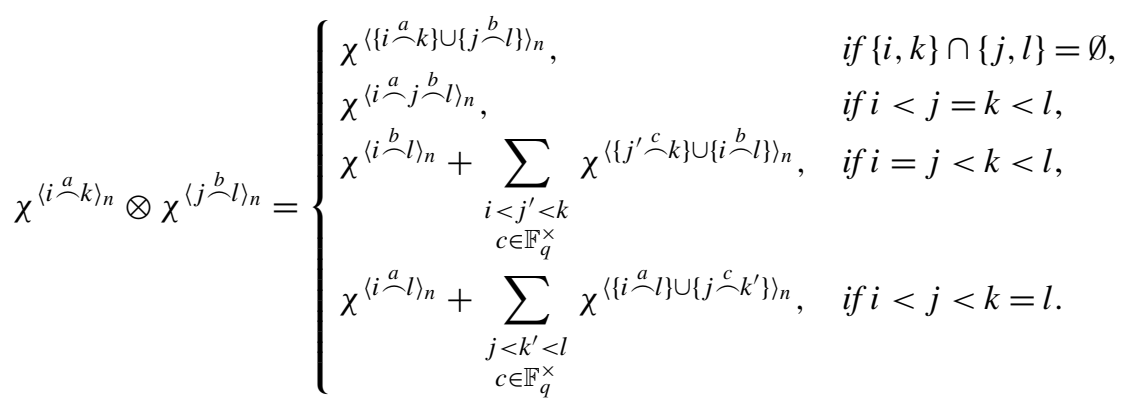

For $i<l, a, b \in \mathbb{F}_{q}^{\times}$,

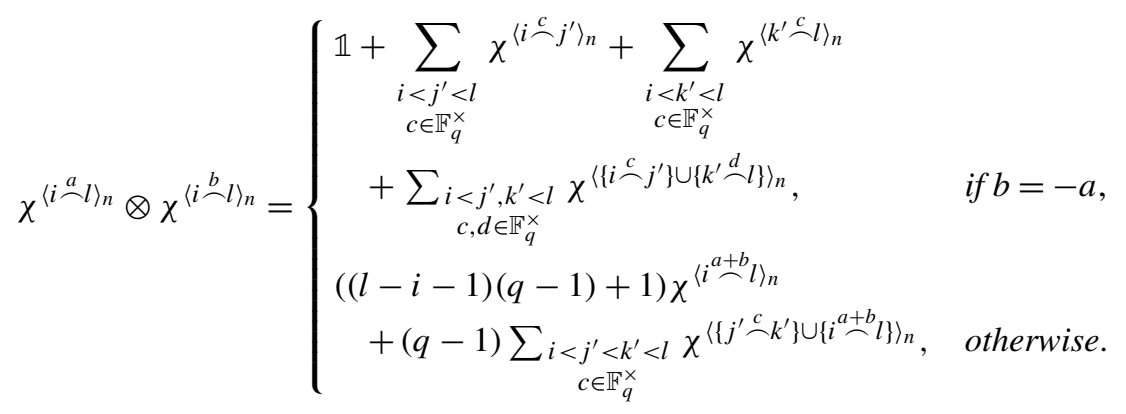

Examples Combinatorially, a product of characters is the superimposition of two setpartition diagrams, such as

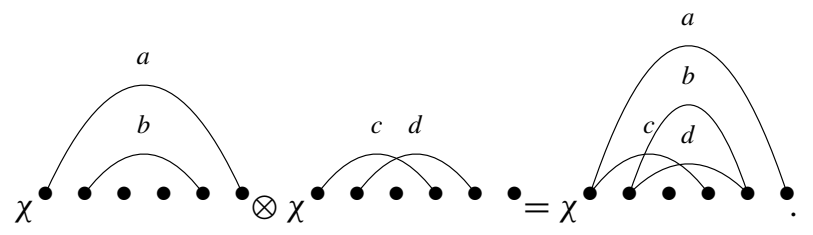


The tensor product rules then describe how to "straighten" the resulting diagram. Usually, we superimpose selectively. For example, since
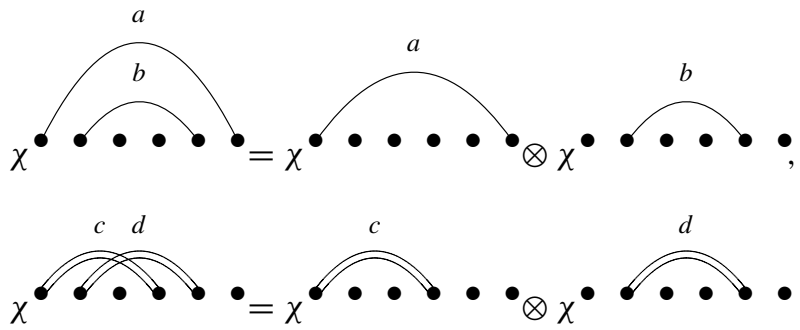

for $d \neq-b$,
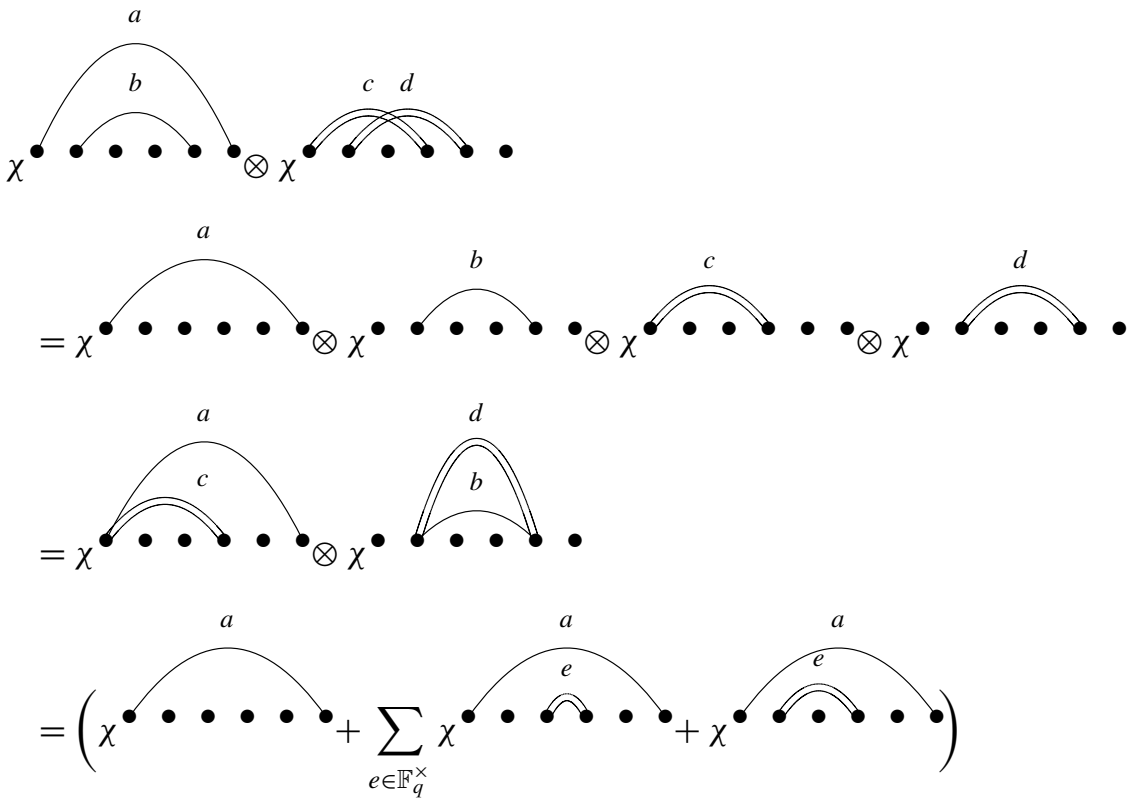

$$
\otimes\left((2 q-1) \chi \bullet \bullet \bullet \bullet+(q-1) \sum_{f \in \mathbb{F}_{q}^{\times}} \chi^{b+d} \bullet \bullet \bullet\right.
$$

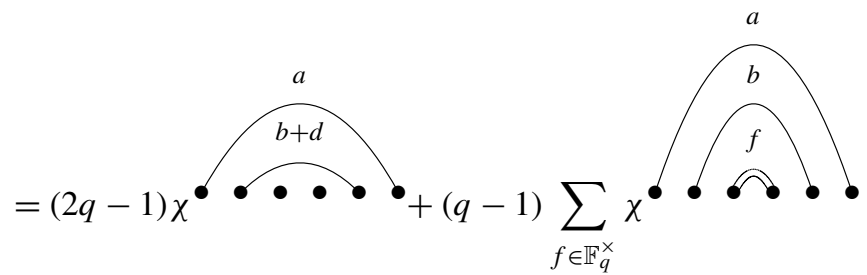



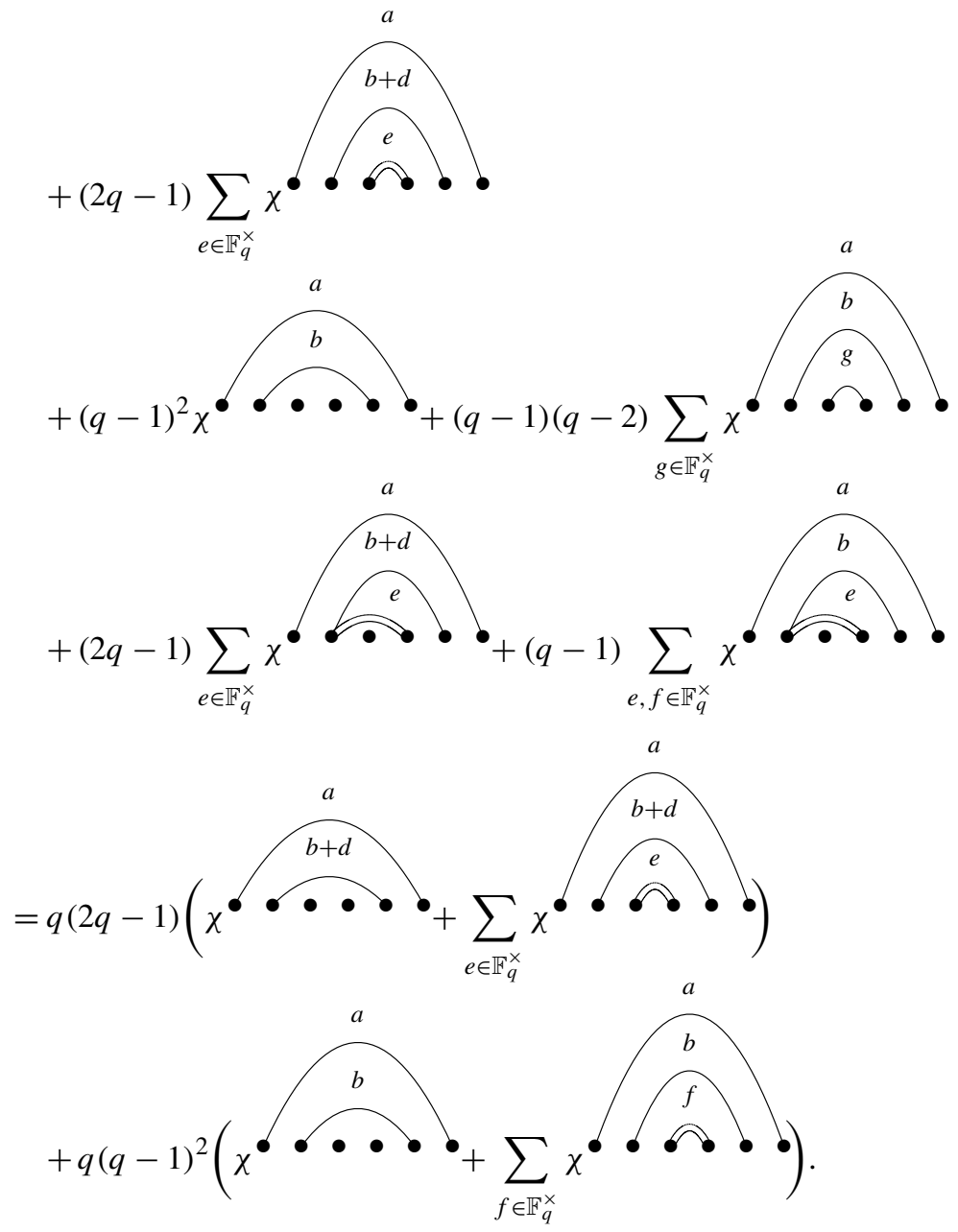

Remark The coefficients of the tensor products are not understood in general, although it is clear from Corollary 4.9 that they are polynomial in $q$.

\subsection{Superinduction}

Let $A \subseteq\{1,2, \ldots, n\}$. If $\mu \in \mathcal{S}_{A}(q)$ and $\lambda \in \mathcal{S}_{n}(q)$, then by Frobenius reciprocity,

$$
\left\langle\chi^{\lambda}, \operatorname{SInd}_{U_{A}}^{U_{n}}\left(\chi^{\mu}\right)\right\rangle_{U_{n}}=\left\langle\operatorname{Res}_{U_{A}}^{U_{n}}\left(\chi^{\lambda}\right), \chi^{\mu}\right\rangle_{U_{A}} .
$$

Thus, if

$$
\operatorname{SInd}_{U_{A}}^{U_{n}}\left(\chi^{\mu}\right)=\sum_{\nu} a_{\mu}^{\nu} \chi^{\nu} \quad \text { and } \quad \operatorname{Res}_{U_{A}}^{U_{n}}\left(\chi^{\lambda}\right)=\sum_{\gamma} b_{\gamma}^{\lambda} \chi^{\gamma},
$$

then by (2.3)

$$
q^{|C(\lambda)|} a_{\mu}^{\lambda}=q^{|C(\mu)|} b_{\mu}^{\lambda},
$$


where $C(v)$ is the set of crossings of $v$. Therefore,

$$
\operatorname{SInd}_{U_{A}}^{U_{n}}\left(\chi^{\mu}\right)=\sum_{\nu} a_{\mu}^{\nu} \chi^{\nu}=\sum_{\nu} q^{|C(\mu)|-|C(\nu)|} b_{\mu}^{\nu} \chi^{\nu}
$$

In general, if $U_{K} \subseteq U_{n}$ with $K \in \mathcal{S}_{n}$, then

$$
\operatorname{SInd}_{U_{K}}^{U_{n}}\left(\chi^{\mu}\right)=\sum_{\nu} q^{\left|C_{K}(\mu)\right|-|C(v)|} b_{\mu}^{v} \chi^{v},
$$

where $C_{K}(v)$ is the set of crossings that occur within the same parts of $K$.

With this discussion, we obtain the following corollary of Sections 4.2 and 4.3. When combined with Corollary 4.14 , below, these results give a reasonably direct way to compute superinduction for some cases.

Corollary 4.10 Let $K=\{1,2, \ldots, k\} \cup\{k+1, k+2, \ldots, n\} \in \mathcal{S}_{n}$ be a set-partition with two parts. Then

$$
\operatorname{SInd}_{U_{K}}^{U_{n}}(\mathbb{1})=\sum_{\begin{array}{c}
\lambda \in \mathcal{S}_{n}(q) \\
\text { if } i \stackrel{j \in \lambda,}{i} \text { then } i \nsim j \in K
\end{array}} q^{-|C(\lambda)|} \chi^{\lambda},
$$

where $i \sim j$ if and only if $i$ and $j$ are in the same part in $K$.

Proof Note that by Theorem 4.6 the coefficient of $\mathbb{1}=\chi^{\bullet \bullet \cdots \bullet}$ in $\operatorname{Res}_{U_{K}}^{U_{n}}\left(\chi^{\lambda}\right)$ is zero unless for every arc $i \frown j \in \mathcal{A}(\lambda)$, the endpoints $i$ and $j$ are in different parts of $K$. Assume that every arc in $\mathcal{A}(\lambda)$ satisfies this condition. Then by Proposition 4.4,

$$
\begin{aligned}
\operatorname{Res}_{U_{K}}^{U_{n}}\left(\chi^{\lambda}\right) & =\left(\bigotimes_{i \frown k \in \lambda} \frac{\operatorname{Res}_{U_{K_{1}}}^{U_{n}}\left(\chi^{\lambda}\right)}{q^{\left|\left\{i<j<k \mid j \notin K_{1}\right\}\right|}}\right) \times\left(\bigotimes_{i \frown k \in \lambda} \frac{\operatorname{Res}_{U_{K_{2}}}^{U_{n}}\left(\chi^{\lambda}\right)}{q^{\left|\left\{i<j<k \mid j \notin K_{2}\right\}\right|}}\right) \\
& =\left(\bigotimes_{i \frown k \in \lambda} \mathbb{1}+\chi_{i, k}^{(1)}\right) \times\left(\bigotimes_{i \frown k \in \lambda} \mathbb{1}+\chi_{i, k}^{(2)}\right),
\end{aligned}
$$

where for $r \in\{1,2\}$,

$$
\chi_{i, k}^{(r)}= \begin{cases}\sum_{\substack{i<j<k, j \in K_{r} \\ a \in \mathbb{F}_{q}^{\times}}} \chi^{j \stackrel{a}{\sim} k}, \quad \text { if } k \in K_{r}, \\ \sum_{\substack{i<j<k, j \in K_{r} \\ a \in \mathbb{F}_{q}^{\times}}} \chi^{i \stackrel{a}{\sim} j}, \quad \text { if } i \in K_{r} .\end{cases}
$$

Thus, the coefficient of $\mathbb{1}$ in $\operatorname{Res}_{U_{K}}^{U_{n}}\left(\chi^{\lambda}\right)$ is 1 . By (4.3), the coefficient of $\chi^{\lambda}$ in $\operatorname{SInd}_{U_{K}}^{U_{n}}(\mathbb{1})$ is $q^{\left|C_{K}(\bullet \bullet \cdots \bullet)\right|-|C(\lambda)|}=q^{-|C(\lambda)|}$. 


\section{Remarks}

(a) This corollary is a specific case. The situation for even general two-part set partitions is more complicated (though seemingly tractable).

(b) Note that the superclass function $\operatorname{SInd}_{U_{K}}^{U_{n}}(\mathbb{1})$ is a linear combination of supercharacters with fractional coefficients, so is a priori not generally a character.

Corollary 4.10 has some immediate combinatorial consequences. Let

$S G_{n \times m}=\left\{a \in M_{n \times n}(\{0,1\}) \mid a\right.$ has at most one 1 and every row and column $\}$

be the set of $m \times n$ 0-1 matrices with at most one 1 in every row and column. Define statistics for $w \in S G_{m \times n}$

$$
\begin{aligned}
\operatorname{ones}(w)= & \left|\left\{(i, j) \in[1, n] \times[1, m] \mid w_{i j}=1\right\}\right| \\
\operatorname{sow}(w)= & \mid\left\{(j, k) \in[1, n] \times[1, m] \mid w_{j k}=0, w_{i k}=1 \text { for some } i<j\right. \text { or } \\
& \left.w_{j l}=1 \text { for some } k<l\right\} \mid .
\end{aligned}
$$

For example, if

$$
w=\left(\begin{array}{cccc}
\underline{0} & 1 & 0 & 0 \\
\underline{0} & \underline{0} & \underline{0} & 1 \\
0 & \underline{0} & 0 & \underline{0}
\end{array}\right), \quad \text { then } \quad \begin{aligned}
& \operatorname{ones}(w)=2 \\
& \operatorname{sow}(w)=6
\end{aligned}
$$

Corollary 4.11 Let $m$ and $n$ be positive integers. Then

(a) $q^{m n}=\sum_{w \in S G_{m \times n}}(q-1)^{\text {ones(w) }} q^{\text {sow }(w)}$.

(b) $0=\sum_{w \in S G_{m \times n}}(-1)^{w_{1 n}}(q-1)^{\text {ones }(w)} q^{\text {sow }(w)}$.

Proof (a) We evaluate the equation in Corollary 4.10 at the identity of $U_{m+n}$, when $K=\{1,2, \ldots, m\} \cup\{m+1, \ldots, m+n\}$. The degree of $\operatorname{SInd}_{U_{K}}^{U_{n}}(\mathbb{1})$ is the size of the factor group $\left|U_{m+n} / U_{K}\right|=q^{m n}$.

On the right-hand side of the equation we sum over the set $X$ of all possible setpartitions whose arcs $i \frown l$ satisfy $i \in\{1, \ldots, m\}$ and $l \in\{m+1, \ldots, m+n\}$. Consider the surjective map $\varphi: X \rightarrow S G_{m \times n}$ given by

$$
\varphi(\lambda)_{i, l-m}=1 \quad \text { if and only if } \quad i \frown l \in \mathcal{A}(\lambda) .
$$

Note that the size of the preimage of $w \in S G_{m \times n}$ is $\left|\varphi^{-1}(w)\right|=(q-1)^{\text {ones }(w)}$. For $\lambda \in X$,

$$
\chi^{\lambda}(1)=\prod_{i \frown l \in \mathcal{A}(\lambda)} q^{l-i-1}=\prod_{i \frown l \in \mathcal{A}(\lambda)} q^{l-m-1+m-i}=q^{\operatorname{sow}(\varphi(\lambda))} q^{|C(\lambda)|},
$$


where each crossing in $C(\lambda)$ accounts for an over-counting of an entry in the computation of $\operatorname{sow}(\varphi(\lambda))$. Thus,

$$
\begin{aligned}
q^{m n}=\operatorname{SInd}_{U_{K}}^{U_{n}}(\mathbb{1})(1) & =\sum_{\substack{\lambda \in \mathcal{S}_{n}(q) \\
\text { if } i \frown j \in \lambda, \\
\text { then } i \nsim j \in K}} q^{-|C(\lambda)|} \chi^{\lambda}(1) \\
& =\sum_{w \in S G_{m \times n}}(q-1)^{\text {ones }(w)} q^{\text {sow }(w)} .
\end{aligned}
$$

(b) Instead of evaluating at the identity, as in (a), evaluate both sides at one of the central non-identity superclasses, each of whose set-partition $\mu$ is of the form $\mu=1 \stackrel{a}{\sim}(m+n)$ for some $a \in \mathbb{F}_{q}^{\times}$.

We conclude with some observations relating superinduction to these superpermutation "characters."

Proposition 4.12 Let $H \subseteq G$ be pattern groups. For a superclass functions $\gamma$ and $\eta$ of $G$,

$$
\left\langle\gamma \otimes \operatorname{SInd}_{H}^{G}(\mathbb{1}), \eta\right\rangle=\left\langle\operatorname{Res}_{H}^{G}(\gamma), \operatorname{Res}_{H}^{G}(\eta)\right\rangle
$$

Proof By a typical Frobenius reciprocity argument,

$$
\begin{aligned}
\left\langle\gamma \otimes \operatorname{SInd}_{H}^{G}(\mathbb{1}), \eta\right\rangle_{G} & =\frac{1}{|G|} \sum_{g \in G} \gamma(g) \operatorname{SInd}_{H}^{G}(\mathbb{1})(g) \overline{\eta(g)} \\
& =\frac{1}{|G|^{2}|H|} \sum_{g, x, y \in G} \gamma(g) \stackrel{\circ}{\mathbb{1}}(x(g-1) y+1) \overline{\eta(g)}
\end{aligned}
$$

where $\mathbb{1}(g)$ is 1 if $g \in H$ and 0 otherwise. By reindexing and using the fact that $\gamma$ and $\eta$ are superclass functions of $G$,

$$
\begin{aligned}
& =\frac{1}{|G|^{2}|H|} \sum_{k, x, y \in G} \gamma\left(x^{-1}(k-1) y^{-1}+1\right) \stackrel{\circ}{\mathbb{1}}(k) \overline{\eta\left(x^{-1}(k-1) y^{-1}+1\right)} \\
& =\frac{1}{|G|^{2}|H|} \sum_{k, x, y \in G} \gamma(k) \stackrel{\circ}{\mathbb{1}}(k) \overline{\eta(k)} \\
& =\frac{1}{|H|} \sum_{k \in H} \gamma(k) \overline{\eta(k)} \\
& =\left\langle\operatorname{Res}_{H}^{G}(\gamma), \operatorname{Res}_{H}^{G}(\eta)\right\rangle_{H} .
\end{aligned}
$$

By choosing $\gamma$ in the above proposition appropriately, we get the following useful corollary via Frobenius reciprocity. 
Corollary 4.13 Let $H \subseteq G$ be pattern groups, and let $\mu \in(H-1)$. If $\chi^{\mu}(1) \times$ $\operatorname{Sinf}_{H}^{G}\left(\chi^{\mu}\right)(h)=\operatorname{Sinf}_{H}^{G}\left(\chi^{\mu}\right)(1) \chi^{\mu}(h)$, for all $h \in H$, then

$$
\operatorname{SInd}_{H}^{G}\left(\chi^{\mu}\right)=\frac{\chi^{\mu}(1)}{\operatorname{Sinf}_{H}^{G}\left(\chi^{\mu}\right)(1)} \operatorname{Sinf}_{H}^{G}\left(\chi^{\mu}\right) \otimes \operatorname{SInd}_{H}^{G}(\mathbb{1}) .
$$

The assumption in Corollary 4.13 is not so unusual. In fact,

Corollary 4.14 Let $U_{K} \subseteq U_{L}$ be parabolic subgroups of $U_{n}$, where $K=K_{1} \cup K_{2} \cup$ $\cdots \cup K_{\ell}, L \in \mathcal{S}_{n}$. Then for $\mu \in \mathcal{S}_{K_{1}}(q) \times \mathcal{S}_{K_{2}}(q) \times \cdots \times \mathcal{S}_{K_{\ell}}(q)$,

$$
\operatorname{SInd}_{U_{K}}^{U_{L}}\left(\chi^{\mu}\right)=\frac{\chi^{\mu}(1)}{\operatorname{Sinf}_{U_{K}}^{U_{L}}\left(\chi^{\mu}\right)(1)} \operatorname{Sinf}_{U_{K}}^{U_{L}}\left(\chi^{\mu}\right) \otimes \operatorname{SInd}_{U_{K}}^{U_{L}}(\mathbb{1}) .
$$

Proof Note that for $i<j \in \mathcal{P}_{K}$ and $k \in U_{K} \subseteq U_{L}$,

$$
\chi^{\langle i \stackrel{a}{\sim} j\rangle_{L}}(k)=\frac{\chi^{\langle i \stackrel{a}{\sim} j\rangle_{L}}(1)}{\chi^{\langle i \stackrel{a}{\sim} j\rangle_{K}(1)}} \chi^{\langle i \stackrel{a}{ } j\rangle_{K}}(k) .
$$

Thus, by the decomposition of characters, for $\mu \in \mathcal{S}_{K_{1}}(q) \times \mathcal{S}_{K_{2}}(q) \times \cdots \times \mathcal{S}_{K_{\ell}}(q)$ and $k \in U_{K}$,

$$
\operatorname{Sinf}_{U_{K}}^{U_{L}}\left(\chi^{\mu}\right)(k)=\chi^{\langle\mu\rangle_{L}}(k)=\frac{\chi^{\langle\mu\rangle_{L}}(1)}{\chi^{\mu}(1)} \chi^{\mu}(k),
$$

and the result now follows from Corollary 4.13.

Remark While the assumption in Corollary 4.13 is sufficient, it is not necessary. For example, if

$$
H=\left\{\left(\begin{array}{lll}
1 & * & * \\
0 & 1 & 0 \\
0 & 0 & 1
\end{array}\right)\right\} \subseteq U_{3}=\left\{\left(\begin{array}{ccc}
1 & * & * \\
0 & 1 & * \\
0 & 0 & 1
\end{array}\right)\right\}
$$

then for these groups,

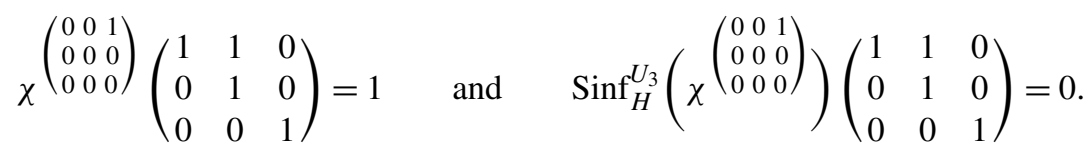

However, it remains true that

$$
\operatorname{SInd}_{H}^{G}\left(\chi\left(\begin{array}{lll}
0 & 0 & 1 \\
0 & 0 & 0 \\
0 & 0 & 0
\end{array}\right)\right)=q^{-1} \operatorname{Sinf}_{H}^{G}\left(\chi\left(\begin{array}{lll}
0 & 0 & 1 \\
0 & 0 & 0 \\
0 & 0 & 0
\end{array}\right)\right) \otimes \operatorname{SInd}_{H}^{G}(\mathbb{1}) .
$$

In fact, the conclusion of Corollary 4.13 may be true for all pattern groups; I know of no counter-example. 
Acknowledgements Much of this work was completed at MSRI during the simultaneous programs "Combinatorial Representation Theory" and "Representation Theory of Finite Groups" in Spring 2008. I especially appreciated related conversations with André, Diaconis, Isaacs, and Yan during this period. I would also like to thank two anonymous referees for their detailed and useful comments.

\section{References}

1. André, C.: Basic characters of the unitriangular group. J. Algebra 175, 287-319 (1995)

2. André, C.: Irreducible characters of finite algebra groups. Matrices and group representations, Coimbra, 1998. Textos Mat. Sér B 19, 65-80 (1999)

3. André, C.: The basic character table of the unitriangular group. J. Algebra 241, 437-471 (2001)

4. André, C.: Basic characters of the unitriangular group (for arbitrary primes). Proc. Am. Math. Soc. 130, 1934-1954 (2002)

5. André, C., Neto, A.: Super-characters of finite unipotent groups of types $B_{n}, C_{n}$ and $D_{n}$. J. Algebra 305, 394-429 (2006)

6. André, C., Nicolás, A.: Supercharacters of the adjoint group of a finite radical ring. J. Group Theory 11, 709-746 (2008)

7. Bergeron, N., Hohlweg, C., Rosas, M., Zabrocki, M.: Grothendieck bialgebras, partition lattices, and symmetric functions in noncommutative variables. Electron. J. Combin. 13(1), Research Paper 75 (2006), 19 pages

8. Bergeron, N., Reutenauer, C., Rosas, M., Zabrocki, M.: Invariants and coinvariants of the symmetric groups in noncommuting variables. Canad. J. Math. 60, 266-296 (2008)

9. Boyarchenko, M., Drinfeld, V.: A motivated introduction to character sheaves and the orbit method for unipotent groups in positive characteristic. arXiv:math/0609769, 2006 preprint

10. Diaconis, P., Isaacs, M.: Supercharacters and superclasses for algebra groups. Trans. Am. Math. Soc. 360, 2359-2392 (2008)

11. Diaconis, P., Thiem, N.: Supercharacter formulas for pattern groups. Trans. Am. Math. Soc. 361, 3501-3533 (2009)

12. Gelfand, I.M., Krob, D., Lascoux, A., Leclerc, B., Retakh, V., Thibon, J.-Y.: Noncommutative symmetric functions. Adv. Math. 112, 218-348 (1995)

13. Hendrickson, A.: Supercharacter theories of finite cyclic groups. Unpublished Ph.D. Thesis, Department of Mathematics, University of Wisconsin, 2008

14. Lewis, S.: Restrictions of supercharacters in $U_{n}\left(\mathbb{F}_{q}\right)$. Unpublished honors thesis, Department of Mathematics, University of Colorado at Boulder, 2009

15. Macdonald, I.G.: Symmetric functions and Hall polynomials, 2nd edn. Oxford Mathematical Monographs. Oxford Science Publications. The Clarendon Press, Oxford University Press, New York (1995). With Contributions by A. Zelevinsky

16. Marberg, E., Thiem, N.: Superinduction for pattern groups. J. Algebra 321, 3681-3703 (2009)

17. Rosas, M., Sagan, B.: Symmetric functions in noncommuting variables. Trans. Am. Math. Soc. 358, 215-232 (2004)

18. Stanley, R.: Enumerative Combinatorics, vol. 2. Cambridge Studies in Advanced Mathematics, vol. 62. Cambridge University Press, Cambridge (1999)

19. Thiem, N., Venkateswaran, V.: Restricting supercharacters of the finite group of unipotent uppertriangular matrices. Electron. J. Combin. 16(1), Research Paper 23 (2009), 32 pages

20. Thiem, N., Vinroot, C.R.: On the characteristic map of finite unitary groups. Adv. Math. 210, 707-732 (2007)

21. Wolf, M.C.: Symmetric functions of noncommuting elements. Duke Math. J. 2, 626-637 (1936)

22. Yan, N.: Representation theory of the finite unipotent linear groups. Unpublished Ph.D. Thesis, Department of Mathematics, University of Pennsylvania, 2001

23. Yan, N.: Representations of finite unipotent linear groups by the method of clusters. 2006 preprint 